Prepared in cooperation with the Sisseton-Wahpeton Oyate

\title{
Pesticide Concentrations in Wetlands on the Lake Traverse Reservation, South and North Dakota, July 2006
}

Open-File Report 2007-1118 


\section{Pesticide Concentrations in Wetlands on the Lake Traverse Reservation, South and North Dakota, July 2006}

By Kathleen M. Neitzert and Roy C. Bartholomay

Prepared in cooperation with the Sisseton-Wahpeton Oyate

Open-File Report 2007-1118 


\section{U.S. Department of the Interior DIRK KEMPTHORNE, Secretary}

\section{U.S. Geological Survey \\ Mark D. Myers, Director}

\section{U.S. Geological Survey, Reston, Virginia: 2007}

For product and ordering information:

World Wide Web: http://www.usgs.gov/pubprod

Telephone: 1-888-ASK-USGS

For more information on the USGS--the Federal source for science about the Earth, its natural and living resources, natural hazards, and the environment:

World Wide Web: http://www.usgs.gov

Telephone: 1-888-ASK-USGS

Any use of trade, product, or firm names is for descriptive purposes only and does not imply endorsement by the U.S. Government.

Although this report is in the public domain, permission must be secured from the individual copyright owners to reproduce any copyrighted materials contained within this report.

Suggested citation:

Neitzert, K.M., and Bartholomay, R.C., 2007, Pesticide concentrations in wetlands on the Lake Traverse Reservation, South and North Dakota, July 2006: U.S. Geological Survey Open-File Report 2007-1118, 25 p. 


\section{Contents}

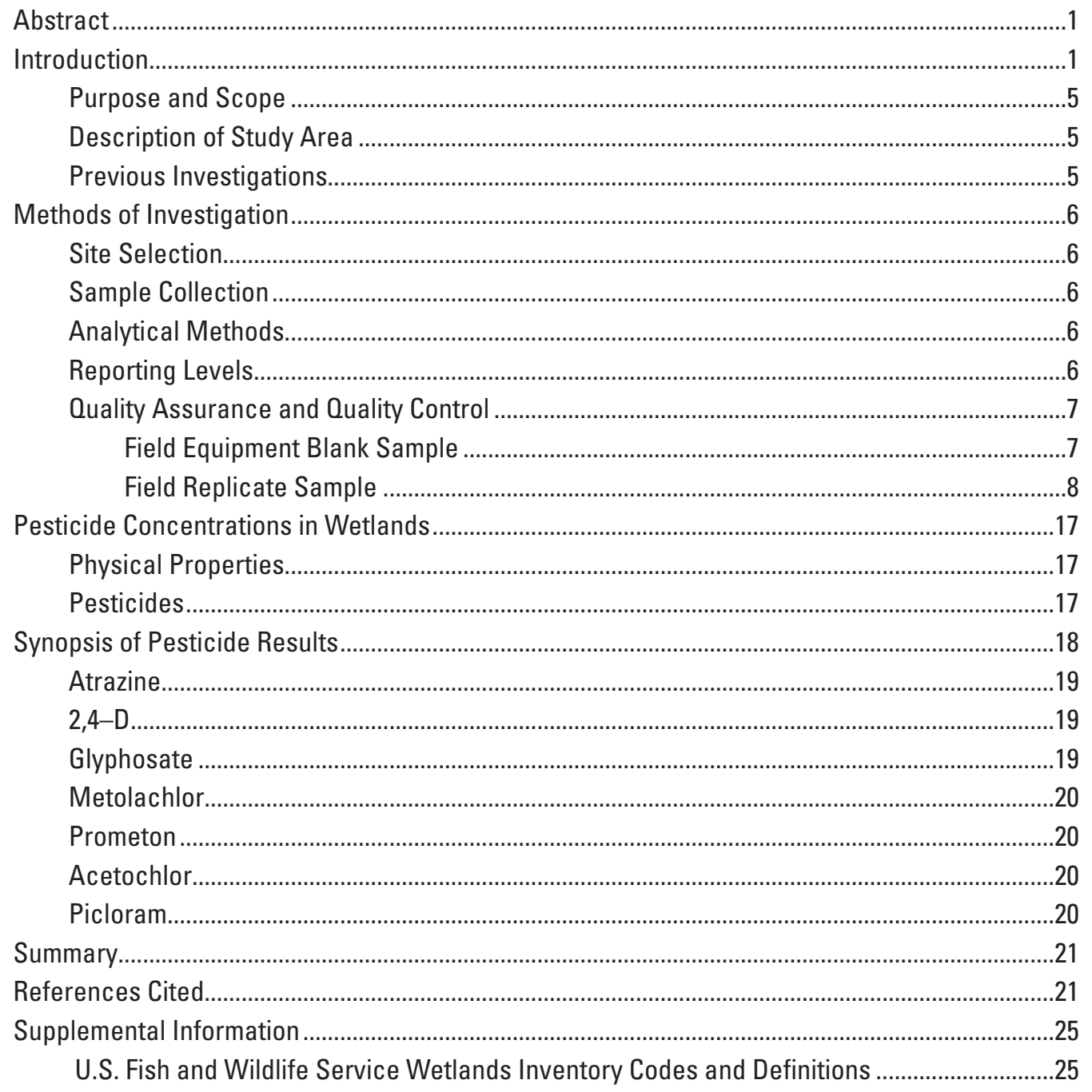

\section{Figures}

1. Map showing location of the historic Lake Traverse Reservation boundry and sample collection sites

2-3. Graphs showing:

2. Number of Lake Traverse Reservation wetlands at which selected pesticides were detected, July 2006.

3. Number of pesticides detected in water samples for selected Lake Traverse Reservation wetlands, July 2006. 


\section{Tables}

1. Estimated use of 34 pesticides in Roberts County, South Dakota, in 1987.

2. Primary wetlands inventory codes for selected Lake Traverse Reservation wetland sampling sites

3. Physical properties of water in selected Lake Traverse Reservation wetlands, July 2006.

4. Pesticide concentrations in water samples from selected Lake Traverse Reservation wetlands, July 2006.

5. U.S. Fish and Wildlife Service wetlands inventory codes for selected Lake Traverse Reservation wetlands at which water-quality samples were collected during July 2006

\section{Conversion Factors, Abbreviations and Acronyms}

\begin{tabular}{lcl} 
Multiply & By & To obtain \\
\hline & Length & \\
\hline inch (in.) & 2.54 & centimeter $(\mathrm{cm})$ \\
inch (in.) & 25.4 & millimeter $(\mathrm{mm})$ \\
foot (ft) & 0.3048 & meter $(\mathrm{m})$ \\
\hline \multicolumn{3}{c}{ Area } \\
\hline acre & 4,047 & square meter $\left(\mathrm{m}^{2}\right)$ \\
acre & 0.4047 & hectare $($ ha) \\
acre & 0.4047 & square hectometer $\left(\mathrm{hm}^{2}\right)$ \\
acre & 0.004047 & hecsquare kilometer $\left(\mathrm{km}^{2}\right)$ \\
square mile $\left(\mathrm{mi}^{2}\right)$ & 2.590 & square kilometer $\left(\mathrm{km}^{2}\right)$ \\
\hline \multicolumn{3}{c}{ Volume } \\
\hline gallon (gal) & 3.785 \\
\hline pound, avoirdupois (lb) & Mass \\
\hline
\end{tabular}

Temperature in degrees Celsius $\left({ }^{\circ} \mathrm{C}\right)$ may be converted to degrees Fahrenheit $\left({ }^{\circ} \mathrm{F}\right)$ as follows:

${ }^{\circ} \mathrm{F}=\left(1.8 x^{\circ} \mathrm{C}\right)+32$

Horizontal coordinate information is referenced to the North American Datum of 1983 (NAD 83).

Specific conductance is given in microsiemens per centimeter at 25 degrees Celsius $(\mu \mathrm{S} / \mathrm{cm}$ at $\left.25^{\circ} \mathrm{C}\right)$.

Concentrations of chemical constituents in water are given either in milligrams per liter (mg/L) or micrograms per liter ( $\mu \mathrm{g} / \mathrm{L})$. 


\section{Abbreviations and Acronyms}

$\begin{array}{ll}< & \text { less than } \\ \text { AMPA } & \text { Aminomethylphosphonic acid } \\ \text { EPTC } & \text { s-Ethyl dipropylcarbomothiate } \\ \text { FNC } & \text { Formazin nephelometric units } \\ \text { LRL } & \text { Laboratory reporting Level } \\ \text { MCL } & \text { Maximum Contaminant Level } \\ \text { MCPA } & \text { (4-Chloro-2-methylphenoxy) acetic acid } \\ \text { MDL } & \text { Method dectection limit } \\ \text { NWIS } & \text { U.S. Geological Survey National Water Information System } \\ \text { NWOL } & \text { U.S. Geological Survey National Water Quality Laboratory } \\ \text { PEMF } & \text { Palustrine, emergent, semipermanently flooded } \\ \text { RPD } & \text { Relative percent difference } \\ \text { SDGS } & \text { South Dakota Department of Environment and Natural Resources, } \\ \text { SWO } & \text { Geological Survey Program } \\ \text { USEPA } & \text { Sisseton-Wahpeton Oyate } \\ \text { USGS } & \text { U.S. Environmental Protection Agency } \\ & \text { U.S. Geological Survey }\end{array}$




\title{
Pesticide Concentrations in Wetlands on the Lake Traverse Reservation, South and North Dakota, July 2006
}

\author{
By Kathleen M. Neitzert and Roy C. Bartholomay
}

\section{Abstract}

During July 2006, water samples were collected from selected Lake Traverse wetlands within the historic Reservation boundary in northeastern South Dakota and southeastern North Dakota as part of a reconnaissancelevel assessment by the U.S. Geological Survey (USGS) and Sisseton-Wahpeton Oyate (SWO). Eighteen wetlands were sampled by the SWO and USGS personnel after primary pesticide application occurred on the Lake Traverse Reservation. Samples were analyzed for 61 pesticide compounds. In addition, the samples were analyzed for physical properties that might help further characterize the water quality of the wetlands.

During July 2006, water samples were collected from 18 wetlands and were analyzed for 61 widely used pesticide compounds. Pesticides were detected in 17 of the 18 sampled wetlands. In the 17 wetlands in which pesticides were detected, the number of pesticides detected ranged from two to eight. Of the 61 pesticides for which the samples were analyzed, 51 pesticides were not detected in any of the wetland samples. Ten pesticides were detected in water samples from at least one of the wetlands at small concentrations. Atrazine and de-ethyl atrazine were detected in samples from 17 of the 18 wetlands. The minimum, maximum, and median concentrations of dissolved atrazine detected were 0.007 , 0.12 , and 0.064 microgram per liter $(\mu \mathrm{g} / \mathrm{L})$, respectively. Two pesticides (picloram and simazine) were detected in only one wetland each. None of the pesticide concentrations exceeded any U.S. Environmental Protection Agency Maximum Contaminant Levels for drinking water, and concentrations were much less than levels considered toxic for humans or aquatic species.

\section{Introduction}

The historic Lake Traverse Reservation boundary encompasses parts of five counties (Roberts, Marshall, Day, Grant, and Codington Counties) in northeastern South Dakota, and parts of two counties (Sargent and Richland Counties) in southeastern North Dakota (fig. 1). The Lake
Traverse Reservation is within the glaciated Central Lowlands physiographic province (Thompson, 2002) and includes parts of two physiographic divisions, the Prairie Coteau and the Minnesota River-Red River lowlands. The Prairie Coteau is a plateau of rolling morainal topography formed by deposition of glacial debris, and the Minnesota River-Red River lowlands is a somewhat flat ground moraine that consists of isolated areas of debris left by retreating glaciers (Thompson, 2002). Some areas are poorly drained and include numerous wetlands that provide a valuable wildlife production resource. Surface water and ground water on the Lake Traverse Reservation are used for public, domestic, stock-water, recreation, and irrigation purposes (Amundson, 2002).

More than 80 percent of the land use within the Lake Traverse Reservation is for grain production, pasture, or hay, and more than 11 percent of the area is covered by surface water in the form of wetlands, lakes, and streams (Thompson, 2002). Pesticides are used mainly to control weeds (herbicides) and insects (insecticides) on crops, although they also may be used to control fungus (fungicides) and nematodes (nematocides) on some crops. Row crops (primarily soybeans and corn) cover about 693 square miles, or about 46 percent of the Lake Traverse Reservation (Thompson, 2002). In 1987, 34 herbicides were used in Roberts County (Battaglin and Goolsby, 1994), with alachlor, atrazine, dicamba, s-ethyl dipropylcarbomothiate (EPTC), bromoxynil, (4-chloro-2-methylphenoxy) acetic acid (MCPA), metolachlor, trifluralin and 2,4-D all applied to more than 10,000 acres of cropland (table 1 ) in that county alone. In 2000, atrazine and metolachlor were the primary herbicides used in Roberts County for corn acreage, and glyphosate was the primary herbicide used for soybean acreage (South Dakota Agricultural Statistics Service, 2001). Although recent pesticide-use data are scarce, it is certain that pesticides are still widely used based on current agricultural practices on the Lake Traverse Reservation.

Pesticides can persist in natural aquatic systems and have long-term effects on biota (Gilliom and others, 2006). Some water-quality pesticide data have been collected by the South Dakota Department of Environment and Natural Resources, Geological Survey Program (SDGS) and the U.S. Geological Survey (USGS) at selected ground-water and surface-water sites, but no data are available for wetlands within the Lake 
Table 1. Estimated use of 34 pesticides in Roberts County, South Dakota, in 1987.

[Data in columns 3, 5, and 9 from Battaglin and Goolsby (1994). Shaded cells indicate pesticides detected in selected Lake Traverse Reservation wetlands (fig. 1). EPTC, s-ethyl dipropylcarbomothiate; MCPA, (4-chloro-2-methylphenoxy) acetic acid; --, not applicable]

\begin{tabular}{|c|c|c|c|c|c|c|c|c|}
\hline Pesticide & $\begin{array}{l}\text { Analyzed for } \\
\text { in selected } \\
\text { Lake Traverse } \\
\text { Reservation } \\
\text { wetlands } \\
\text { (table 4) }\end{array}$ & $\begin{array}{l}\text { Acres }^{1} \\
\text { treated }\end{array}$ & $\begin{array}{c}\text { Rank } \\
\text { acres } \\
\text { treated }\end{array}$ & $\begin{array}{l}\text { Pounds }^{2} \\
\text { of active } \\
\text { ingredient } \\
\text { applied }\end{array}$ & $\begin{array}{c}\text { Rank of } \\
\text { active } \\
\text { ingredient } \\
\text { applied (all } \\
\text { pesticides) }\end{array}$ & $\begin{array}{l}\text { Rank of active } \\
\text { ingredient } \\
\text { applied } \\
\text { for pesticides } \\
\text { analyzed for } \\
\text { in samples } \\
\text { from Lake } \\
\text { Traverse } \\
\text { Reservation } \\
\text { wetlands }\end{array}$ & $\begin{array}{c}\text { Pounds }^{3} \\
\text { applied } \\
\text { per treated } \\
\text { acre }\end{array}$ & $\begin{array}{l}\text { Pounds }^{4} \\
\text { used per } \\
\text { square mile } \\
\text { of Roberts } \\
\text { County }\end{array}$ \\
\hline $2,4-\mathrm{D}$ & Yes & 67,614 & 1 & 25,029 & 5 & 4 & 2.701 & 22.281 \\
\hline 2,4-DB & No & 2 & 33 & 2 & 33 & -- & 1.000 & .002 \\
\hline Acifluorfen & No & 2,645 & 23 & 661 & 24 & -- & 4.002 & .588 \\
\hline Bentazon & No & 9,523 & 10 & 7,999 & 10 & -- & 1.191 & 7.121 \\
\hline Bromoxynil & No & 14,734 & 7 & 4,289 & 16 & -- & 3.435 & 3.818 \\
\hline Butylate & Yes & 1,299 & 27 & 5,196 & 13 & 9 & .250 & 4.625 \\
\hline Chloramben & No & 1,444 & 25 & 3,417 & 19 & -- & .423 & 3.042 \\
\hline Chlorsulfuron & No & 4,356 & 19 & 86 & 29 & -- & 50.651 & .077 \\
\hline Cyanazine & No & 5,845 & 15 & 6,956 & 12 & -- & .840 & 6.192 \\
\hline Dalapon & No & 48 & 31 & 36 & 32 & -- & 1.333 & .032 \\
\hline EPTC & No & 16,469 & 6 & 72,185 & 1 & -- & .228 & 64.258 \\
\hline Ethalfluralin & Yes & 4,861 & 18 & 4,571 & 15 & 11 & 1.063 & 4.069 \\
\hline
\end{tabular}


Table 1. Estimated use of 34 pesticides in Roberts County, South Dakota, in 1987.—Continued

[Data in columns 3, 5, and 9 from Battaglin and Goolsby (1994). Shaded cells indicate pesticides detected in selected Lake Traverse Reservation wetlands (fig. 1). EPTC, s-ethyl dipropylcarbomothiate; MCPA, (4-chloro-2-methylphenoxy) acetic acid; --, not applicable]

\begin{tabular}{|c|c|c|c|c|c|c|c|c|}
\hline Pesticide & $\begin{array}{l}\text { Analyzed for } \\
\text { in selected } \\
\text { Lake Traverse } \\
\text { Reservation } \\
\text { wetlands } \\
\text { (table 4) }\end{array}$ & $\begin{array}{l}\text { Acres }^{1} \\
\text { treated }\end{array}$ & $\begin{array}{c}\text { Rank } \\
\text { acres } \\
\text { treated }\end{array}$ & $\begin{array}{l}\text { Pounds }^{2} \\
\text { of active } \\
\text { ingredient } \\
\text { applied }\end{array}$ & $\begin{array}{c}\text { Rank of } \\
\text { active } \\
\text { ingredient } \\
\text { applied (all } \\
\text { pesticides) }\end{array}$ & $\begin{array}{l}\text { Rank of active } \\
\text { ingredient } \\
\text { applied } \\
\text { for pesticides } \\
\text { analyzed for } \\
\text { in samples } \\
\text { from Lake } \\
\text { Traverse } \\
\text { Reservation } \\
\text { wetlands }\end{array}$ & $\begin{array}{l}\text { Pounds }^{3} \\
\text { applied } \\
\text { per treated } \\
\text { acre }\end{array}$ & $\begin{array}{l}\text { Pounds }^{4} \\
\text { used per } \\
\text { square mile } \\
\text { of Roberts } \\
\text { County }\end{array}$ \\
\hline Fluazifop & No & 529 & 30 & 106 & 28 & -- & 4.991 & 0.094 \\
\hline Glyphosate & Yes & 9,187 & 12 & 4,957 & 14 & 10 & 1.853 & 4.413 \\
\hline Imazethapyr & No & 5,290 & 16 & 265 & 25 & -- & 19.962 & .236 \\
\hline Metribuzin & Yes & 3,403 & 21 & 1,040 & 22 & 14 & 3.272 & .926 \\
\hline Metsulfuron & No & 6,725 & 13 & 67 & 31 & -- & 100.373 & .060 \\
\hline Pendimethalin & Yes & 6,015 & 14 & 7,314 & 11 & 8 & .822 & 6.511 \\
\hline Picloram & Yes & 1,984 & 24 & 992 & 23 & 15 & 2.000 & .883 \\
\hline Propachlor & Yes & 3,251 & 22 & 16,254 & 6 & 5 & .200 & 14.469 \\
\hline Sethoxydim & No & 1,058 & 28 & 212 & 26 & -- & 4.991 & .189 \\
\hline Simazine & Yes & 758 & 29 & 1,137 & 20 & 13 & .667 & 1.012 \\
\hline
\end{tabular}

${ }^{1}$ Rounded to the nearest acre.

${ }^{2}$ Rounded to the nearest pound.

${ }^{3}$ Calculated from columns 3 and 5 .

${ }^{4}$ Rounded to three decimal places. 


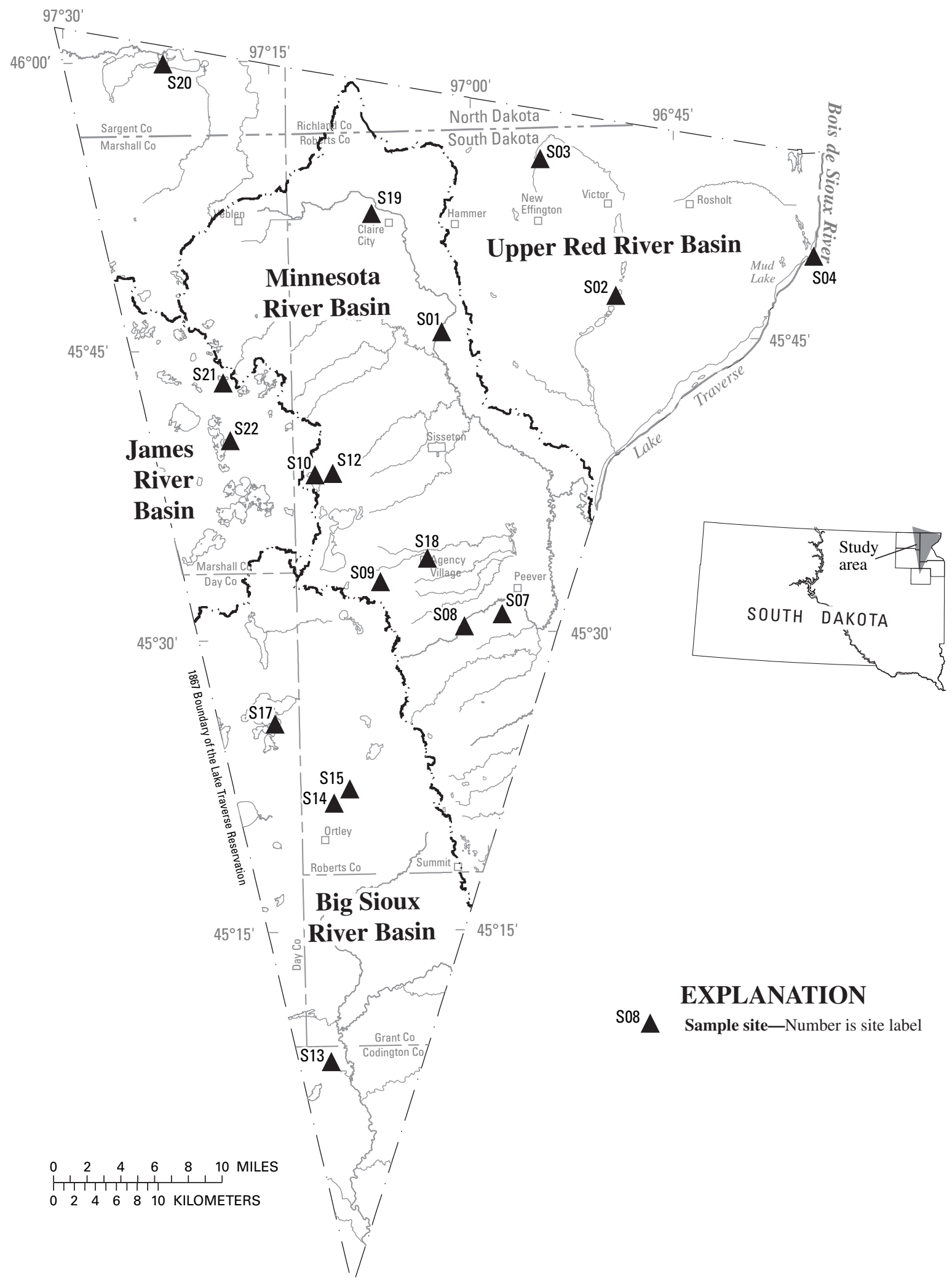

Base from U.S. Geological Survey digital line graph 1:100,000, 1993 Universal Transverse Mercator projection, zone 14

Figure 1. Location of the historic Lake Traverse Reservation boundry and sample collection sites. 
Traverse Reservation. Previously collected water-quality data indicate that several pesticides, including alachlor, atrazine, and 2,4-D, have been detected at concentrations greater than laboratory reporting levels (LRLs) (Thompson, 2000, 2002). Some of the pesticides that are applied on the Lake Traverse Reservation (including alachlor and atrazine) are known endocrine disruptors (Danzo, 1997; Wilson and others, 1996; and Rawlings and others, 1998), whereas others (including glyphosate and 2,4-D) have various levels of toxicity to aquatic organisms (U.S. Forest Service, 1995, 1997).

To address concerns about the quality of water in Lake Traverse Reservation wetlands, the USGS, in cooperation with the Sisseton-Wahpeton Oyate (SWO), conducted a reconnaissance-level study of pesticide concentrations in selected wetlands. The samples were analyzed for 61 widely used pesticides. The locations of the selected Lake Traverse Reservation wetlands that were sampled for pesticides are shown in figure 1 .

\section{Purpose and Scope}

The purpose of this report is to present the results of a reconnaissance-level assessment of pesticide concentrations in selected Lake Traverse Reservation wetlands during July 2006. Specifically, this report presents (1) a summary of sample collection and analytical methods; (2) concentrations of pesticides and physical properties for selected Lake Traverse Reservation wetlands; and (3) a synopsis of the pesticide results for those pesticides detected at concentrations greater than LRLs. Analytical results for water samples include physical properties and pesticide concentrations for 18 wetlands. The quality of water in all Lake Traverse Reservation wetlands cannot be thoroughly characterized with such a limited sampling program; however, the results may be useful to assess the effects of land-management practices on the aquatic resources and to identify possible water-quality concerns that could be the focus of more comprehensive investigations in the future.

\section{Description of Study Area}

The study area includes the area within the historic 1867 boundary of the Lake Traverse Reservation (hereafter called the Lake Traverse Reservation) and much of Roberts County, and parts of Marshall, Day, Grant, and Codington Counties in northeastern South Dakota, and parts of Sargent and Richland Counties in southeastern North Dakota (fig. 1). The Lake Traverse Reservation has an area of about 1,508 square miles. The Bois des Sioux River, Lake Traverse, and Mud Lake form most of the northeastern boundary of Roberts County.

Numerous lakes, ponds, and wetlands are located in the Lake Traverse Reservation, particularly within internally drained, noncontributing areas of the basins of the Big
Sioux and James Rivers. Many of the lakes are hydraulically connected to glacial-outwash aquifers, and lake levels tend to rise or fall with the water table (Lawrence, 1989). Several of the larger lakes within the study area have been developed for recreation, whereas many of the smaller lakes and wetlands are used for livestock watering or wildlife-production areas (Thompson, 2001).

The study area is relatively sparsely populated, with a population in Roberts County of 10,016 in 2000 (U.S. Census Bureau, 2001). Municipalities and rural water systems currently provide most of the water used in the study area, with nearly all of it from ground-water sources. Surface-water use is limited to livestock watering (Thompson, 2001).

\section{Previous Investigations}

Although little information is available about pesticide concentrations in wetlands within the Lake Traverse Reservation, some previous investigations were useful in developing the study described in this report. A comprehensive water-resources investigation for the Lake Traverse Reservation was completed by Thompson (2001). As part of that investigation, samples from 13 observation wells were analyzed for atrazine, carbofuran, cyanazine, and 2,4-D using enzyme-linked immunosorbent assay analysis; a sample from one well had a detectable concentration of 2,4-D (Thompson, 2000).

Additional water-quality analyses for pesticides from other USGS studies in the Lake Traverse Reservation area were compiled from the USGS National Water Information System (NWIS) database. A total of 113 pesticides and pesticide metabolites were analyzed in samples from two wells and six surface-water sites; six pesticides were detected. Lindane was detected in four samples, and 2,4-D, dicamba, dieldrin, heptachlor, and p, $\mathrm{p}^{\prime}-\mathrm{DDE}$ were detected in one sample each (Thompson, 2002, table 4).

The SDGS has periodically sampled two surface-water sites and nine ground-water sites. Atrazine, 2,4-D, and alachlor were detected at the surface-water sites, and simazine was detected at one ground-water site (Thompson, 2002, tables 2-3).

A reconnaissance-level assessment of pesticides in wetlands on the Yankton Sioux Reservation in southeastern South Dakota was completed by Schaap and Bartholomay (2006). Samples from 19 wetlands were analyzed for 61 pesticide compounds, and 13 pesticides were detected in at least one wetland. Samples from all 19 wetlands contained at least three to eight pesticides.

As part of a reconnaissance-level assessment of organic wastewater compounds in the Upper Big Sioux River Basin (Sando and others, 2005), samples from the Big Sioux River were analyzed for three pesticides (atrazine, metolachlor, and prometon). All three pesticides were detected in some of the samples. 


\section{Methods of Investigation}

This study was designed to provide a reconnaissancelevel assessment of pesticide concentrations in selected wetlands within the Lake Traverse Reservation. Waterquality samples were collected for pesticide analyses from 18 wetlands during July 2006. Site selection, sample collection, analytical methods, reporting levels, and quality assurance and quality control are described in this section.

\section{Site Selection}

Samples were collected from 18 wetlands on the Lake Traverse Reservation (fig. 1). Sites were selected to best represent the broad range of wetlands found within the Lake Traverse Reservation including physical location, size, and type (as indicated by the U.S. Fish and Wildlife National Wetlands Inventory; table 2). The area received above-normal precipitation in April 2006 (National Weather Service, 2006a, 2006b), and crop planting began later than normal. Due to the wet conditions, the normal spring pesticide application was finished later than normal, requiring the pesticide sampling to be completed in mid-summer. The wetlands from which samples were collected were fairly well distributed throughout the study area (fig. 1). The area of the sampled wetlands ranged from a few acres to a few hundred acres. The amount of vegetation in and surrounding the wetlands ranged from little (primarily dirt) to extreme (heavy cattails, bushes, or trees), dependent on location and type of wetland.

Sampled wetlands were classified using wetlands inventory codes (U.S. Fish and Wildlife Service, 2004). These alpha-numeric codes were developed to correspond to the classification nomenclature that best describes the habitat. In some cases, the sampled wetlands had been assigned multiple codes to note multiple influences on the wetlands (table 2). For these sites, the code assigned for the largest area of the wetland where the sample was collected was considered to be the primary code for this report, and varying codes for smaller areas associated with the primary area were considered to be additional influence codes. A primary wetlands inventory code had been assigned to 16 of the sampled wetlands. Of the wetlands with primary codes, four also had multiple class codes designated within the wetland area. The two sites with no assigned primary code did have additional influence codes noted. The most frequently noted code was PEMF (Palustrine, Emergent, Semipermanently Flooded). More information on the wetland codes is given in the Supplemental Information Section at the back of this report.

\section{Sample Collection}

Eighteen wetlands were sampled by SWO and USGS personnel in July 2006 after primary pesticide application occurred on the Lake Traverse Reservation. In general, sampling procedures followed guidelines described by Wilde and others (1999) using two-person, ultra-clean procedures, but these procedures were adapted to the conditions encountered at the different sites. For each sampled wetland, this process involved some combination of collecting water in bottles, directly or after filtering, or collecting several liters of water in a large container and later transferring it to the appropriate bottles in a mobile laboratory. For determination of dissolved pesticides, filtering was performed using precleaned, baked, glass-fiber 142-millimeter filters or precleaned 0.7-micron glass fiber filters. Samples for total pesticides were containerized in pre-cleaned, 1-liter, glass amber bottles. Samples were collected by wading when possible; when the depth or bottom material of the wetland did not allow wading, a boat was used. All samples were grab samples collected at one vertical location. All samples were chilled according to sample specifications and then packed for overnight shipping to the USGS National Water Quality Laboratory (NWQL) located in Denver, Colorado, for analyses.

\section{Analytical Methods}

Standard methods were used to analyze samples for physical properties (information on specific analytical methods can be accessed at http://nwql.usgs.gov/Public/ref_ list.html). Samples for pesticides were analyzed at the NWQL. Analyses for the 61 pesticides were performed using methods described in Wershaw and others (1987), Zaugg and others (1995), and Lee and others (2001).

\section{Reporting Levels}

The presence of some constituents, even at very low concentrations, is of interest because of potential health effects of the constituents. Childress and others (1999) provides details about the approach used by the USGS regarding detection levels and reporting levels.

The method detection limit (MDL) is the minimum concentration of a substance that can be measured and reported with 99-percent confidence that the concentration is greater than zero. The laboratory reporting level (LRL) is the concentration at which the false negative error rate is minimized to be no more than one percent of the reported results. The LRL generally is equal to twice the yearly determined long-term MDL, which is a detection level derived by determining the standard deviation of a minimum of 24 MDL spike-sample measurements over an extended time. The long-term MDL controls false positive error and is the concentration at which the false positive risk is minimized to be no more than one percent of the reported values (Childress and others, 1999). These reporting levels may be described as preliminary for a developmental method if the levels have been based on a small number of analytical results. Also, these levels may vary from sample to sample for the same 
Table 2. Primary wetlands inventory codes for selected Lake Traverse Reservation wetland sampling sites.

\begin{tabular}{|c|c|c|c|c|}
\hline $\begin{array}{c}\text { Site } \\
\text { (fig. 1) }\end{array}$ & Station number & $\begin{array}{l}\text { Primary wetlands } \\
\text { inventory code } \\
\text { (table 5) }\end{array}$ & Basin land use & $\begin{array}{c}\text { Approximate } \\
\text { wetland size or area } \\
\text { sampled within wetland } \\
\text { (in acres) }\end{array}$ \\
\hline${ }^{1} \mathrm{~S} 01$ & 454535097023100 & PFOC & Crops, grazing & 36 \\
\hline S02 & 454717096493000 & L2ABG & Grazing & 50 \\
\hline S03 & 455426096545100 & PEMF & Crops & 8 \\
\hline S04 & 454933096361200 & $\mathrm{PEM} / \mathrm{ABFh}$ & Marsh, crops & 170 \\
\hline S07 & 453107096583500 & PEMF & Marsh, crops & 18 \\
\hline S08 & 453031097011800 & $\mathrm{PAB} / \mathrm{EMF}$ & Crops & 9 \\
\hline S09 & 453245097071800 & PEMFx & Crops & 8 \\
\hline S10 & 453801097120100 & L1UBG & Crops, marsh, grazing & 200 \\
\hline $\mathrm{S} 12$ & 453825097104400 & PEMC & Grazing & 1 \\
\hline S13 & 450811097114200 & PEMF & Crops, grazing & 6 \\
\hline S14 & 452129097111500 & PEM/SSB & Crops, grazing & 100 \\
\hline S15 & 452214097095900 & None noted & Crops grazing & 19 \\
\hline S17 & 452540097142000 & L2ABG & Unused & 75 \\
\hline${ }^{2} \mathrm{~S} 18$ & 453354097040800 & None noted & Unused, in town & 8 \\
\hline S19 & 455145097072900 & PEMF & Marsh, crops & 6 \\
\hline S20 & 455941097223800 & $\mathrm{PEM} / \mathrm{ABF}$ & Unused, refuge & 2 \\
\hline S21 & 454315097184400 & PEM/ABF & Tribal grazing & 9 \\
\hline S22 & 454012097181700 & PABF & Tribal grazing & 4 \\
\hline
\end{tabular}

${ }^{1}$ Field equipment blank sample collected at site.

${ }^{2}$ Replicate sample collected at site.

constituent and the same method if matrix effects or other factors arise that interfere with the analysis. Concentrations measured between the MDL and the LRL are described as estimated values. For most of the constituents in this report, reported concentrations are greater than the LRLs unless they are indicated as estimated. An estimated concentration should be considered a qualitatively detected analyte (Childress and others, 1999).

\section{Quality Assurance and Quality Control}

Analytical results should describe the environmental conditions at the time the samples were collected.

Unfortunately, certain factors such as sampling error can affect the process and ultimately lead to analytical results that are not representative of the natural conditions. Several techniques, collectively known as quality assurance and quality control, were used to evaluate the precision and accuracy of the reported analytical results for this study. Quality-assurance samples collected during July 2006 as part of this study included one field equipment blank sample and one replicate sample. Analytical results for the quality-assurance samples are presented in the tables along with the analytical results for the environmental samples.

\section{Field Equipment Blank Sample}

The field equipment blank sample was used to assess the possible contamination of samples or analytical error. The blank sample was collected by passing ultra-pure water through the collection and processing equipment used for environmental samples. Procedures used were identical to those used to collect and process the environmental samples. 
The blank sample was collected at the same location as one of the environmental samples and just before the environmental sample was collected. If no contamination of the sample is introduced during the collection, processing, transport, and analysis of the blank sample, and the analytical results are accurate, then none of the constituents should be detected.

A field blank was collected at wetland site S01 on July 11, 2006 (tables 3-4). All the pesticide concentrations in the blank sample were less than the LRLs, indicating the sampling and analytical methods did not contaminate the samples or produce false detections.

\section{Field Replicate Sample}

A field replicate sample is intended to be an exact copy of the environmental sample. The field replicate sample was collected and processed immediately after the associated primary environmental sample was collected and processed, and procedures used for the replicate sample were identical to those used for the primary sample. Any differences in the analytical results between the environmental sample and the replicate sample may indicate some combination of inconsistency of sample collection, the natural variability in the sampled water, and the variability of the analytical method.
A field replicate was collected at wetland site $\mathrm{S} 18$ on July 13, 2006 (tables 3-4). Precision of analytical results for the field replicate sample was determined by calculating the relative percent difference (RPD) for the primary/replicate sample pair as indicated in the following equation:

$$
R P D=(d / \bar{x}) * 100
$$

where

$$
\begin{aligned}
d= & \text { difference in concentration between the } \\
& \text { primary environmental sample and the } \\
& \text { field replicate sample for a given primary/ } \\
& \text { replicate sample pair, and } \\
\bar{x}= & \text { mean concentration of the primary } \\
& \text { environmental sample and the field } \\
& \text { replicate sample for a given primary/ } \\
& \text { replicate pair. }
\end{aligned}
$$

All the primary/replicate sample pairs either had concentrations less than the LRL for both pairs or had the given constituent detected in both sample pairs (table 3). All the RPDs were within the acceptable level of precision, which generally is 20 percent or less (Taylor, 1987). 
Table 3. Physical properties of water in selected Lake Traverse Reservation wetlands, July 2006.

[Location of sites shown in figure 1. mm of $\mathrm{Hg}$, millimeters of mercury; FNU, formazin nephelometric turbidity units; mg/L, milligrams per liter; $\mu \mathrm{S} / \mathrm{cm}$, microsiemens per centimeter at 25 degrees Celsius; deg C, degrees Celsius; --, no data. Number in parentheses below property is U.S. Geological Survey National Water Information System parameter code]

\begin{tabular}{|c|c|c|c|c|c|c|c|c|c|c|c|}
\hline Site & Station number & $\begin{array}{c}\text { Date } \\
\text { (mm-dd-yyyy) }\end{array}$ & Time & $\begin{array}{l}\text { Water depth } \\
\text { (meters) } \\
\text { (82903) }\end{array}$ & $\begin{array}{c}\text { Barometric } \\
\text { pressure } \\
(\mathrm{mm} \text { of } \mathrm{Hg}) \\
(00025)\end{array}$ & $\begin{array}{c}\text { Turbidity } \\
\text { (FNU) } \\
\text { (63680) }\end{array}$ & $\begin{array}{c}\begin{array}{c}\text { Oxygen, } \\
\text { dissolved } \\
(\mathrm{mg} / \mathrm{L}) \\
(00300)\end{array}\end{array}$ & $\begin{array}{c}\text { pH, } \\
\text { whole field, } \\
\text { standard } \\
\text { units } \\
(00400)\end{array}$ & $\begin{array}{c}\text { Specific } \\
\text { conductance, } \\
\mu \mathrm{S} / \mathrm{cm} \\
(00095)\end{array}$ & $\begin{array}{c}\text { Temperature, } \\
\text { water } \\
\text { (deg C) } \\
(\mathbf{0 0 0 1 0 )}\end{array}$ & $\begin{array}{c}\text { Temperature, } \\
\text { air } \\
(\text { deg C) } \\
(00020)\end{array}$ \\
\hline S01 & 454535097023100 & 07-11-2006 & 0910 & 1.27 & 729 & -- & 2.0 & 7.8 & 897 & 19.9 & 25.0 \\
\hline S02 & 454717096493000 & 07-12-2006 & 0930 & 1.28 & 733 & 6.1 & 8.3 & 8.5 & 1,490 & 24.8 & 25.0 \\
\hline S03 & 455426096545100 & 07-11-2006 & 1355 & 1.22 & 732 & 16 & 7.0 & 8.2 & 995 & 26.0 & 33.0 \\
\hline S04 & 454933096361200 & 07-12-2006 & 1130 & .21 & 736 & 6.1 & 8.4 & 8.7 & 1,400 & 27.3 & 30.0 \\
\hline S07 & 453107096583500 & 07-20-2006 & 0950 & .06 & 736 & 65 & 3.5 & 8.1 & 5,040 & 18.8 & 25.0 \\
\hline S08 & 453031097011800 & 07-20-2006 & 0910 & .27 & 730 & 66 & 9.2 & 8.8 & 1,360 & 24.1 & 22.0 \\
\hline S09 & 453245097071800 & 07-13-2006 & 1030 & .61 & 703 & 1.0 & 3.0 & 7.4 & 320 & 20.5 & 25.0 \\
\hline S10 & 453801097120100 & 07-12-2006 & 1440 & 4.88 & 709 & 3.5 & 9.2 & 8.8 & 681 & 24.5 & 32.0 \\
\hline S12 & 453825097104400 & 07-12-2006 & 1540 & .15 & 712 & 230 & 18.5 & 8.7 & 409 & 32.7 & 34.0 \\
\hline $\mathrm{S} 13$ & 450811097114200 & 07-20-2006 & 1255 & .12 & 720 & 160 & 4.0 & 6.7 & 775 & 19.3 & 38.0 \\
\hline S14 & 452129097111500 & 07-20-2006 & 1135 & .31 & 719 & 1.5 & 4.6 & 7.4 & 552 & 18.1 & 34.0 \\
\hline S15 & 452214097095900 & 07-20-2006 & 1105 & .17 & 718 & 5.3 & 5.0 & 7.4 & 502 & 20.2 & 25.0 \\
\hline S17 & 452540097142000 & 07-20-2006 & 1040 & .46 & 718 & .5 & 11.9 & 9.4 & 823 & 24.8 & 24.0 \\
\hline S18 & 453354097040800 & 07-13-2006 & 1120 & 1.22 & 717 & 3.2 & 10.3 & 8.5 & 1,240 & 25.7 & 26.0 \\
\hline${ }^{1} \mathrm{~S} 18$ & 453354097040800 & 07-13-2006 & 1130 & 1.22 & 717 & 3.2 & 10.3 & 8.5 & 1,240 & 25.7 & 26.0 \\
\hline S19 & 455145097072900 & 07-11-2006 & 1025 & .76 & 729 & 34 & 1.4 & 7.0 & 885 & 20.0 & 31.0 \\
\hline S20 & 455941097223800 & 07-11-2006 & 1240 & .70 & 728 & 20 & 2.2 & 7.7 & 1,360 & 19.4 & 29.0 \\
\hline$S 21$ & 454315097184400 & 07-13-2006 & 0850 & .34 & 706 & 3.8 & 4.8 & 8.2 & 1,230 & 23.3 & 24.5 \\
\hline S22 & 454012097181700 & 07-13-2006 & 0930 & .46 & 707 & 1.0 & 7.2 & 9.0 & 272 & 23.7 & 25.0 \\
\hline
\end{tabular}

${ }^{1}$ Replicate sample collected at site. 
[Location of sites shown in figure 1. All concentrations in micrograms per liter. All concentrations are dissolved, unless otherwise noted. RPD, relative percent difference; AMPA, aminomethylphosphonic acid; EPTC, s-ethyl dipropylcarbomothiate; <, less than; E, estimated. Shading represents estimated values or values greater than the reporting level. Number in parentheses below constituent is

U.S. Geological Survey National Water Information System parameter code]

\begin{tabular}{|c|c|c|c|c|c|c|c|c|c|c|c|c|}
\hline Site & Station number & $\begin{array}{c}\text { Date } \\
\text { (mm-dd-yyyy) }\end{array}$ & Time & $\begin{array}{c}2,4,5-T, \\
\text { total } \\
(39740)\end{array}$ & $\begin{array}{c}\text { 2,4-D, } \\
\text { total } \\
(39730)\end{array}$ & $\begin{array}{c}\text { 2,6-Di- } \\
\text { ethyl } \\
\text { aniline } \\
\text { (82660) }\end{array}$ & $\begin{array}{c}\text { Aceto- } \\
\text { chlor } \\
(49260)\end{array}$ & $\begin{array}{c}\text { Alachlor } \\
\text { (46342) }\end{array}$ & $\begin{array}{c}\text { alpha- } \\
\text { HCH } \\
(34253)\end{array}$ & $\begin{array}{l}\text { AMPA } \\
(62649)\end{array}$ & $\begin{array}{c}\text { Atrazine } \\
\text { (39632) }\end{array}$ & $\begin{array}{c}\text { Azinphos- } \\
\text { methyl } \\
\text { (82686) }\end{array}$ \\
\hline S01 & 454535097023100 & 07-11-2006 & 0910 & $<0.03$ & 0.11 & $<0.006$ & $<0.006$ & $<0.005$ & $<0.005$ & 0.46 & 0.11 & $<0.050$ \\
\hline${ }^{1} \mathrm{~S} 01$ & 454535097023100 & 07-11-2006 & 0900 & $<.03$ & $<.02$ & $<.006$ & $<.006$ & $<.005$ & $<.005$ & $<.310$ & $<.007$ & $<.050$ \\
\hline $\mathrm{S} 02$ & 454717096493000 & 07-12-2006 & 0930 & $<.03$ & $<.02$ & $<.006$ & $<.006$ & $<.005$ & $<.005$ & $<.310$ & .033 & $<.050$ \\
\hline S03 & 455426096545100 & 07-11-2006 & 1355 & $<.03$ & $<.02$ & $<.006$ & $<.006$ & $<.005$ & $<.005$ & $<.310$ & .052 & $<.050$ \\
\hline S04 & 454933096361200 & $07-12-2006$ & 1130 & $<.03$ & .03 & $<.006$ & $<.006$ & $<.005$ & $<.005$ & E. 260 & .12 & $<.050$ \\
\hline S07 & 453107096583500 & 07-20-2006 & 0950 & $<.03$ & $<.02$ & $<.006$ & $<.006$ & $<.005$ & $<.005$ & $<.310$ & .025 & $<.050$ \\
\hline S08 & 453031097011800 & 07-20-2006 & 0910 & $<.03$ & E.11 & $<.006$ & $<.006$ & $<.005$ & $<.005$ & E. 130 & .094 & $<.050$ \\
\hline S09 & 453245097071800 & 07-13-2006 & 1030 & $<.03$ & .1 & $<.006$ & $<.006$ & $<.005$ & $<.005$ & $<.310$ & .076 & $<.050$ \\
\hline $\mathrm{S} 10$ & 453801097120100 & 07-12-2006 & 1440 & $<.03$ & .1 & $<.006$ & E.007 & $<.005$ & $<.005$ & $<.310$ & .05 & $<.050$ \\
\hline $\mathrm{S} 12$ & 453825097104400 & 07-12-2006 & 1540 & $<.03$ & $<.02$ & $<.006$ & $<.006$ & $<.005$ & $<.005$ & $<.310$ & .068 & $<.050$ \\
\hline $\mathrm{S} 13$ & 450811097114200 & 07-20-2006 & 1255 & $<.03$ & E.11 & $<.006$ & $<.006$ & $<.005$ & $<.005$ & $<.310$ & .021 & $<.050$ \\
\hline $\mathrm{S} 14$ & 452129097111500 & 07-20-2006 & 1135 & $<.03$ & $<.02$ & $<.006$ & $<.006$ & $<.005$ & $<.005$ & $<.310$ & $<.007$ & $<.050$ \\
\hline $\mathrm{S} 15$ & 452214097095900 & 07-20-2006 & 1105 & $<.03$ & $<.02$ & $<.006$ & $<.006$ & $<.005$ & $<.005$ & $<.310$ & .007 & $<.050$ \\
\hline S17 & 452540097142000 & 07-20-2006 & 1040 & $<.03$ & E.05 & $<.006$ & $<.006$ & $<.005$ & $<.005$ & $<.310$ & .079 & $<.050$ \\
\hline S18 & 453354097040800 & 07-13-2006 & 1120 & $<.03$ & .03 & $<.006$ & E.008 & $<.005$ & $<.005$ & $<.310$ & .037 & $<.050$ \\
\hline${ }^{2} \mathrm{~S} 18$ & 453354097040800 & 07-13-2006 & 1130 & $<.03$ & .03 & $<.006$ & E.009 & $<.005$ & $<.005$ & $<.310$ & .039 & $<.050$ \\
\hline S19 & 455145097072900 & 07-11-2006 & 1025 & $<.03$ & $<.02$ & $<.006$ & $<.006$ & $<.005$ & $<.005$ & E. 150 & .013 & $<.050$ \\
\hline S20 & 455941097223800 & 07-11-2006 & 1240 & $<.03$ & $<.02$ & $<.006$ & $<.006$ & $<.005$ & $<.005$ & E.090 & .096 & $<.050$ \\
\hline $\mathrm{S} 21$ & 454315097184400 & 07-13-2006 & 0850 & $<.03$ & $<.02$ & $<.006$ & $<.006$ & $<.005$ & $<.005$ & $<.310$ & .064 & $<.050$ \\
\hline $\mathrm{S} 22$ & 454012097181700 & 07-13-2006 & 0930 & $<.03$ & .08 & $<.006$ & $<.006$ & $<.005$ & $<.005$ & $<.310$ & .064 & $<.050$ \\
\hline$R P D^{3}$ & & & & 0 & 0 & 0 & 11.8 & 0 & 0 & 0 & 5.3 & 0 \\
\hline
\end{tabular}


Table 4. Pesticide concentrations in water samples from selected Lake Traverse Reservation wetlands, July 2006.—Continued

[Location of sites shown in figure 1. All concentrations in micrograms per liter. All concentrations are dissolved, unless otherwise noted. RPD, relative percent difference; AMPA, aminomethylphosphonic acid; EPTC, s-ethyl dipropylcarbomothiate; <, less than; E, estimated. Shading represents estimated values or values greater than the reporting level. Number in parentheses below constituent is U.S. Geological Survey National Water Information System parameter code]

\begin{tabular}{|c|c|c|c|c|c|c|c|c|c|c|c|c|}
\hline Site & Station number & $\begin{array}{c}\text { Date } \\
\text { (mm-dd-yyyy) }\end{array}$ & Time & $\begin{array}{c}\text { Ben- } \\
\text { fluralin } \\
\text { (82673) }\end{array}$ & $\begin{array}{c}\text { Butylate } \\
\text { (04028) }\end{array}$ & $\begin{array}{c}\text { Carbaryl } \\
\text { (82680) }\end{array}$ & $\begin{array}{c}\text { Carbo- } \\
\text { furan } \\
(82674)\end{array}$ & $\begin{array}{l}\text { Chlor- } \\
\text { pyrifos } \\
(38933)\end{array}$ & $\begin{array}{c}\text { cis- } \\
\text { Perme- } \\
\text { thrin } \\
(\mathbf{8 2 6 8 7})\end{array}$ & $\begin{array}{l}\text { Cyan- } \\
\text { azine } \\
\text { (04041) }\end{array}$ & $\begin{array}{c}\text { DCPA } \\
(82682)\end{array}$ & $\begin{array}{c}\text { De-ethyl } \\
\text { atrazine } \\
(04040)\end{array}$ \\
\hline S01 & 454535097023100 & 07-11-2006 & 0910 & $<0.010$ & $<0.004$ & $<0.041$ & $<0.020$ & $<0.005$ & $<0.006$ & $<0.018$ & $<0.003$ & E0.029 \\
\hline${ }^{1} \mathrm{~S} 01$ & 454535097023100 & 07-11-2006 & 0900 & $<.010$ & $<.004$ & $<.041$ & $<.020$ & $<.005$ & $<.006$ & $<.018$ & $<.003$ & $<.014$ \\
\hline $\mathrm{S} 02$ & 454717096493000 & 07-12-2006 & 0930 & $<.010$ & $<.004$ & $<.041$ & $<.020$ & $<.005$ & $<.006$ & $<.018$ & $<.003$ & E.011 \\
\hline S03 & 455426096545100 & 07-11-2006 & 1355 & $<.010$ & $<.004$ & $<.041$ & $<.020$ & $<.005$ & $<.006$ & $<.018$ & $<.003$ & E.014 \\
\hline S04 & 454933096361200 & 07-12-2006 & 1130 & $<.010$ & $<.004$ & $<.041$ & $<.020$ & $<.005$ & $<.006$ & $<.018$ & $<.003$ & E.028 \\
\hline S07 & 453107096583500 & 07-20-2006 & 0950 & $<.010$ & $<.004$ & $<.041$ & $<.020$ & $<.005$ & $<.006$ & $<.018$ & $<.003$ & E.010 \\
\hline S08 & 453031097011800 & 07-20-2006 & 0910 & $<.010$ & $<.004$ & $<.041$ & $<.020$ & $<.005$ & $<.006$ & $<.018$ & $<.003$ & E.017 \\
\hline S09 & 453245097071800 & 07-13-2006 & 1030 & $<.010$ & $<.004$ & $<.041$ & $<.020$ & $<.005$ & $<.006$ & $<.018$ & $<.003$ & E.023 \\
\hline $\mathrm{S} 10$ & 453801097120100 & $07-12-2006$ & 1440 & $<.010$ & $<.004$ & $<.041$ & $<.020$ & $<.005$ & $<.006$ & $<.018$ & $<.003$ & E.017 \\
\hline $\mathrm{S} 12$ & 453825097104400 & 07-12-2006 & 1540 & $<.010$ & $<.004$ & $<.041$ & $<.020$ & $<.005$ & $<.006$ & $<.018$ & $<.003$ & E.030 \\
\hline $\mathrm{S} 13$ & 450811097114200 & 07-20-2006 & 1255 & $<.010$ & $<.004$ & $<.041$ & $<.020$ & $<.005$ & $<.006$ & $<.018$ & $<.003$ & E.007 \\
\hline $\mathrm{S} 14$ & 452129097111500 & 07-20-2006 & 1135 & $<.010$ & $<.004$ & $<.041$ & $<.020$ & $<.005$ & $<.006$ & $<.018$ & $<.003$ & $<.014$ \\
\hline $\mathrm{S} 15$ & 452214097095900 & 07-20-2006 & 1105 & $<.010$ & $<.004$ & $<.041$ & $<.020$ & $<.005$ & $<.006$ & $<.018$ & $<.003$ & E.005 \\
\hline S17 & 452540097142000 & 07-20-2006 & 1040 & $<.010$ & $<.004$ & $<.041$ & $<.020$ & $<.005$ & $<.006$ & $<.018$ & $<.003$ & E.025 \\
\hline S18 & 453354097040800 & 07-13-2006 & 1120 & $<.010$ & $<.004$ & $<.041$ & $<.020$ & $<.005$ & $<.006$ & $<.018$ & $<.003$ & E.011 \\
\hline${ }^{2} \mathrm{~S} 18$ & 453354097040800 & 07-13-2006 & 1130 & $<.010$ & $<.004$ & $<.041$ & $<.020$ & $<.005$ & $<.006$ & $<.018$ & $<.003$ & E.012 \\
\hline S19 & 455145097072900 & 07-11-2006 & 1025 & $<.010$ & $<.004$ & $<.041$ & $<.020$ & $<.005$ & $<.006$ & $<.018$ & $<.003$ & E.007 \\
\hline S20 & 455941097223800 & 07-11-2006 & 1240 & $<.010$ & $<.004$ & $<.041$ & $<.020$ & $<.005$ & $<.006$ & $<.018$ & $<.003$ & E.028 \\
\hline $\mathrm{S} 21$ & 454315097184400 & 07-13-2006 & 0850 & $<.010$ & $<.004$ & $<.041$ & $<.020$ & $<.005$ & $<.006$ & $<.018$ & $<.003$ & E.022 \\
\hline $\mathrm{S} 22$ & 454012097181700 & 07-13-2006 & 0930 & $<.010$ & $<.004$ & $<.041$ & $<.020$ & $<.005$ & $<.006$ & $<.018$ & $<.003$ & E.021 \\
\hline$R P D^{3}$ & & & & 0 & 0 & 0 & 0 & 0 & 0 & 0 & 0 & 8.7 \\
\hline
\end{tabular}


[Location of sites shown in figure 1. All concentrations in micrograms per liter. All concentrations are dissolved, unless otherwise noted. RPD, relative percent difference; AMPA, aminomethylphosphonic acid; EPTC, s-ethyl dipropylcarbomothiate; <, less than; E, estimated. Shading represents estimated values or values greater than the reporting level. Number in parentheses below constituent is

U.S. Geological Survey National Water Information System parameter code]

\begin{tabular}{|c|c|c|c|c|c|c|c|c|c|c|c|c|}
\hline Site & Station number & $\begin{array}{c}\text { Date } \\
\text { (mm-dd-yyyy) }\end{array}$ & Time & $\begin{array}{l}\text { Desulfinyl- } \\
\text { fipronil } \\
(62170)\end{array}$ & $\begin{array}{c}\text { Desulfinyl- } \\
\text { fipronil- } \\
\text { amide } \\
\text { (62169) }\end{array}$ & $\begin{array}{c}\text { Diazinon } \\
\text { (39572) }\end{array}$ & $\begin{array}{c}\text { Dicamba, } \\
\text { total } \\
(82052)\end{array}$ & $\begin{array}{c}\text { Dichlor- } \\
\text { prop, } \\
\text { total } \\
(82183)\end{array}$ & $\begin{array}{l}\text { Dieldrin } \\
\text { (39381) }\end{array}$ & $\begin{array}{l}\text { Disulfoton } \\
\text { (82677) }\end{array}$ & $\begin{array}{c}\text { EPTC } \\
(82668)\end{array}$ & $\begin{array}{l}\text { Ethal- } \\
\text { fluralin } \\
\text { (82663) }\end{array}$ \\
\hline S01 & 454535097023100 & 07-11-2006 & 0910 & $<0.012$ & $<0.029$ & $<0.005$ & $<0.03$ & $<0.02$ & $<0.009$ & $<0.02$ & $<0.004$ & $<0.009$ \\
\hline${ }^{1} \mathrm{~S} 01$ & 454535097023100 & 07-11-2006 & 0900 & $<.012$ & $<.029$ & $<.005$ & $<.03$ & $<.02$ & $<.009$ & $<.02$ & $<.004$ & $<.009$ \\
\hline $\mathrm{S} 02$ & 454717096493000 & 07-12-2006 & 0930 & $<.012$ & $<.029$ & $<.005$ & $<.03$ & $<.02$ & $<.009$ & $<.02$ & $<.004$ & $<.009$ \\
\hline $\mathrm{S} 03$ & 455426096545100 & 07-11-2006 & 1355 & $<.012$ & $<.029$ & $<.005$ & $<.03$ & $<.02$ & $<.009$ & $<.02$ & $<.004$ & $<.009$ \\
\hline S04 & 454933096361200 & 07-12-2006 & 1130 & $<.012$ & $<.029$ & $<.005$ & $<.03$ & $<.02$ & $<.009$ & $<.02$ & $<.004$ & $<.009$ \\
\hline S07 & 453107096583500 & 07-20-2006 & 0950 & $<.012$ & $<.029$ & $<.005$ & $<.03$ & $<.02$ & $<.009$ & $<.02$ & $<.004$ & $<.009$ \\
\hline S08 & 453031097011800 & 07-20-2006 & 0910 & $<.012$ & $<.029$ & $<.005$ & $<.03$ & $<.02$ & $<.009$ & $<.02$ & $<.004$ & $<.009$ \\
\hline S09 & 453245097071800 & 07-13-2006 & 1030 & $<.012$ & $<.029$ & $<.005$ & $<.03$ & $<.02$ & $<.009$ & $<.02$ & $<.004$ & $<.009$ \\
\hline $\mathrm{S} 10$ & 453801097120100 & 07-12-2006 & 1440 & $<.012$ & $<.029$ & $<.005$ & $<.03$ & $<.02$ & $<.009$ & $<.02$ & $<.004$ & $<.009$ \\
\hline $\mathrm{S} 12$ & 453825097104400 & $07-12-2006$ & 1540 & $<.012$ & $<.029$ & $<.005$ & $<.03$ & $<.02$ & $<.009$ & $<.02$ & $<.004$ & $<.009$ \\
\hline $\mathrm{S} 13$ & 450811097114200 & 07-20-2006 & 1255 & $<.012$ & $<.029$ & $<.013$ & $<.03$ & $<.02$ & $<.009$ & $<.02$ & $<.004$ & $<.009$ \\
\hline $\mathrm{S} 14$ & 452129097111500 & 07-20-2006 & 1135 & $<.012$ & $<.029$ & $<.005$ & $<.03$ & $<.02$ & $<.009$ & $<.02$ & $<.004$ & $<.009$ \\
\hline $\mathrm{S} 15$ & 452214097095900 & 07-20-2006 & 1105 & $<.012$ & $<.029$ & $<.005$ & $<.03$ & $<.02$ & $<.009$ & $<.02$ & $<.004$ & $<.009$ \\
\hline $\mathrm{S} 17$ & 452540097142000 & 07-20-2006 & 1040 & $<.012$ & $<.029$ & $<.005$ & $<.03$ & $<.02$ & $<.009$ & $<.02$ & $<.004$ & $<.009$ \\
\hline $\mathrm{S} 18$ & 453354097040800 & $07-13-2006$ & 1120 & $<.012$ & $<.029$ & $<.005$ & $<.03$ & $<.02$ & $<.009$ & $<.02$ & $<.004$ & $<.009$ \\
\hline${ }^{2} \mathrm{~S} 18$ & 453354097040800 & $07-13-2006$ & 1130 & $<.012$ & $<.029$ & $<.005$ & $<.03$ & $<.02$ & $<.009$ & $<.02$ & $<.004$ & $<.009$ \\
\hline S19 & 455145097072900 & 07-11-2006 & 1025 & $<.012$ & $<.029$ & $<.005$ & $<.03$ & $<.02$ & $<.009$ & $<.02$ & $<.004$ & $<.009$ \\
\hline $\mathrm{S} 20$ & 455941097223800 & $07-11-2006$ & 1240 & $<.012$ & $<.029$ & $<.005$ & $<.03$ & $<.02$ & $<.009$ & $<.02$ & $<.004$ & $<.009$ \\
\hline $\mathrm{S} 21$ & 454315097184400 & 07-13-2006 & 0850 & $<.012$ & $<.029$ & $<.005$ & $<.03$ & $<.02$ & $<.009$ & $<.02$ & $<.004$ & $<.009$ \\
\hline $\mathrm{S} 22$ & 454012097181700 & 07-13-2006 & 0930 & $<.012$ & $<.029$ & $<.005$ & $<.03$ & $<.02$ & $<.009$ & $<.02$ & $<.004$ & $<.009$ \\
\hline$R P D^{3}$ & & & & 0 & 0 & 0 & 0 & 0 & 0 & 0 & 0 & 0 \\
\hline
\end{tabular}


Table 4. Pesticide concentrations in water samples from selected Lake Traverse Reservation wetlands, July 2006.—Continued

[Location of sites shown in figure 1. All concentrations in micrograms per liter. All concentrations are dissolved, unless otherwise noted. RPD, relative percent difference; AMPA, aminomethylphosphonic acid; EPTC, s-ethyl dipropylcarbomothiate; <, less than; E, estimated. Shading represents estimated values or values greater than the reporting level. Number in parentheses below constituent is U.S. Geological Survey National Water Information System parameter code]

\begin{tabular}{|c|c|c|c|c|c|c|c|c|c|c|c|c|}
\hline Site & Station number & $\begin{array}{c}\text { Date } \\
\text { (mm-dd-yyyy) }\end{array}$ & Time & $\begin{array}{l}\text { Ethoprop } \\
(82672)\end{array}$ & $\begin{array}{c}\text { Fipronil } \\
(62166)\end{array}$ & $\begin{array}{l}\text { Fipronil } \\
\text { sulfide } \\
\text { (62167) }\end{array}$ & $\begin{array}{l}\text { Fipronil } \\
\text { sulfone } \\
(62168)\end{array}$ & $\begin{array}{c}\text { Fonofos } \\
\text { (04095) }\end{array}$ & $\begin{array}{c}\text { Glufosinate } \\
\text { (62721) }\end{array}$ & $\begin{array}{c}\text { Gly- } \\
\text { phosate } \\
\text { (62722) }\end{array}$ & $\begin{array}{l}\text { Lindane } \\
\text { (39341) }\end{array}$ & $\begin{array}{r}\text { Linuron } \\
\text { (82666) }\end{array}$ \\
\hline S01 & 454535097023100 & 07-11-2006 & 0910 & $<0.012$ & $<0.016$ & $<0.013$ & $<0.024$ & $<0.005$ & $<0.140$ & $<0.150$ & $<0.004$ & $<0.035$ \\
\hline${ }^{1} \mathrm{~S} 01$ & 454535097023100 & 07-11-2006 & 0900 & $<.012$ & $<.016$ & $<.013$ & $<.024$ & $<.005$ & $<.140$ & $<.150$ & $<.004$ & $<.035$ \\
\hline S02 & 454717096493000 & 07-12-2006 & 0930 & $<.012$ & $<.016$ & $<.013$ & $<.024$ & $<.005$ & $<.140$ & E.060 & $<.004$ & $<.035$ \\
\hline S03 & 455426096545100 & 07-11-2006 & 1355 & $<.012$ & $<.016$ & $<.013$ & $<.024$ & $<.005$ & $<.140$ & $<.150$ & $<.004$ & $<.035$ \\
\hline S04 & 454933096361200 & 07-12-2006 & 1130 & $<.012$ & $<.016$ & $<.013$ & $<.024$ & $<.005$ & $<.140$ & E. 070 & $<.004$ & $<.035$ \\
\hline S07 & 453107096583500 & 07-20-2006 & 0950 & $<.012$ & $<.016$ & $<.013$ & $<.024$ & $<.005$ & $<.140$ & $<.150$ & $<.004$ & $<.035$ \\
\hline S08 & 453031097011800 & 07-20-2006 & 0910 & $<.012$ & $<.016$ & $<.013$ & $<.024$ & $<.005$ & $<.140$ & $<.150$ & $<.004$ & $<.035$ \\
\hline S09 & 453245097071800 & 07-13-2006 & 1030 & $<.012$ & $<.016$ & $<.013$ & $<.024$ & $<.005$ & $<.140$ & E.050 & $<.004$ & $<.035$ \\
\hline $\mathrm{S} 10$ & 453801097120100 & 07-12-2006 & 1440 & $<.012$ & $<.016$ & $<.013$ & $<.024$ & $<.005$ & $<.140$ & $<.150$ & $<.004$ & $<.035$ \\
\hline $\mathrm{S} 12$ & 453825097104400 & 07-12-2006 & 1540 & $<.012$ & $<.016$ & $<.013$ & $<.024$ & $<.005$ & $<.140$ & $<.150$ & $<.004$ & $<.035$ \\
\hline S13 & 450811097114200 & 07-20-2006 & 1255 & $<.012$ & $<.016$ & $<.013$ & $<.024$ & $<.005$ & $<.140$ & E. 110 & $<.004$ & $<.035$ \\
\hline S14 & 452129097111500 & 07-20-2006 & 1135 & $<.012$ & $<.016$ & $<.013$ & $<.024$ & $<.005$ & $<.140$ & $<.150$ & $<.004$ & $<.035$ \\
\hline S15 & 452214097095900 & 07-20-2006 & 1105 & $<.012$ & $<.016$ & $<.013$ & $<.024$ & $<.005$ & $<.140$ & $<.150$ & $<.004$ & $<.035$ \\
\hline S17 & 452540097142000 & 07-20-2006 & 1040 & $<.012$ & $<.016$ & $<.013$ & $<.024$ & $<.005$ & $<.140$ & $<.150$ & $<.004$ & $<.035$ \\
\hline S18 & 453354097040800 & 07-13-2006 & 1120 & $<.012$ & $<.016$ & $<.013$ & $<.024$ & $<.005$ & $<.140$ & $<.150$ & $<.004$ & $<.035$ \\
\hline${ }^{2} \mathrm{~S} 18$ & 453354097040800 & 07-13-2006 & 1130 & $<.012$ & $<.016$ & $<.013$ & $<.024$ & $<.005$ & $<.140$ & $<.150$ & $<.004$ & $<.035$ \\
\hline S19 & 455145097072900 & 07-11-2006 & 1025 & $<.012$ & $<.016$ & $<.013$ & $<.024$ & $<.005$ & $<.140$ & $<.150$ & $<.004$ & $<.035$ \\
\hline $\mathrm{S} 20$ & 455941097223800 & 07-11-2006 & 1240 & $<.012$ & $<.016$ & $<.013$ & $<.024$ & $<.005$ & $<.140$ & .3 & $<.015$ & $<.035$ \\
\hline $\mathrm{S} 21$ & 454315097184400 & 07-13-2006 & 0850 & $<.012$ & $<.016$ & $<.013$ & $<.024$ & $<.005$ & $<.140$ & $<.150$ & $<.008$ & $<.035$ \\
\hline $\mathrm{S} 22$ & 454012097181700 & 07-13-2006 & 0930 & $<.012$ & $<.016$ & $<.013$ & $<.024$ & $<.005$ & $<.140$ & $<.150$ & $<.004$ & $<.035$ \\
\hline$R P D^{3}$ & & & & 0 & 0 & 0 & 0 & 0 & 0 & 0 & 0 & 0 \\
\hline
\end{tabular}


[Location of sites shown in figure 1. All concentrations in micrograms per liter. All concentrations are dissolved, unless otherwise noted. RPD, relative percent difference; AMPA, aminomethylphosphonic acid; EPTC, s-ethyl dipropylcarbomothiate; <, less than; E, estimated. Shading represents estimated values or values greater than the reporting level. Number in parentheses below constituent is

U.S. Geological Survey National Water Information System parameter code]

\begin{tabular}{|c|c|c|c|c|c|c|c|c|c|c|c|c|}
\hline Site & Station number & $\begin{array}{c}\text { Date } \\
\text { (mm-dd-yyyy) }\end{array}$ & Time & $\begin{array}{c}\text { Malathion } \\
\text { (39532) }\end{array}$ & $\begin{array}{l}\text { Meto- } \\
\text { lachlor } \\
\text { (39415) }\end{array}$ & $\begin{array}{c}\text { Metribuzin } \\
\text { (82630) }\end{array}$ & $\begin{array}{c}\text { Molinate } \\
\text { (82671) }\end{array}$ & $\begin{array}{c}\text { Naprop- } \\
\text { amide } \\
(82684)\end{array}$ & $\begin{array}{c}\text { p,p'-DDE } \\
(34653)\end{array}$ & $\begin{array}{c}\text { Parathion } \\
\text { (39542) }\end{array}$ & $\begin{array}{c}\text { Methyl } \\
\text { parathion } \\
(82667) \\
\end{array}$ & $\begin{array}{c}\text { Pebulate } \\
\text { (82669) }\end{array}$ \\
\hline S01 & 454535097023100 & 07-11-2006 & 0910 & $<0.027$ & $<0.006$ & $<0.028$ & $<0.003$ & $<0.007$ & $<0.003$ & $<0.010$ & $<0.015$ & $<0.004$ \\
\hline${ }^{1} \mathrm{~S} 01$ & 454535097023100 & $07-11-2006$ & 0900 & $<.027$ & $<.006$ & $<.028$ & $<.003$ & $<.007$ & $<.003$ & $<.010$ & $<.015$ & $<.004$ \\
\hline S02 & 454717096493000 & 07-12-2006 & 0930 & $<.027$ & $<.006$ & $<.028$ & $<.003$ & $<.007$ & $<.003$ & $<.010$ & $<.015$ & $<.004$ \\
\hline S03 & 455426096545100 & $07-11-2006$ & 1355 & $<.027$ & $<.006$ & $<.028$ & $<.003$ & $<.007$ & $<.003$ & $<.010$ & $<.015$ & $<.004$ \\
\hline S04 & 454933096361200 & $07-12-2006$ & 1130 & $<.027$ & .027 & $<.028$ & $<.003$ & $<.007$ & $<.003$ & $<.010$ & $<.015$ & $<.004$ \\
\hline S07 & 453107096583500 & 07-20-2006 & 0950 & $<.027$ & $<.006$ & $<.028$ & $<.003$ & $<.007$ & $<.003$ & $<.010$ & $<.015$ & $<.008$ \\
\hline S08 & 453031097011800 & 07-20-2006 & 0910 & $<.027$ & .008 & $<.028$ & $<.003$ & $<.007$ & $<.003$ & $<.010$ & $<.015$ & $<.004$ \\
\hline S09 & 453245097071800 & 07-13-2006 & 1030 & $<.027$ & $<.006$ & $<.028$ & $<.003$ & $<.007$ & $<.003$ & $<.010$ & $<.015$ & $<.004$ \\
\hline $\mathrm{S} 10$ & 453801097120100 & 07-12-2006 & 1440 & $<.027$ & $<.006$ & $<.028$ & $<.003$ & $<.007$ & $<.003$ & $<.010$ & $<.015$ & $<.004$ \\
\hline $\mathrm{S} 12$ & 453825097104400 & $07-12-2006$ & 1540 & $<.027$ & $<.006$ & $<.028$ & $<.003$ & $<.007$ & $<.003$ & $<.010$ & $<.015$ & $<.034$ \\
\hline $\mathrm{S} 13$ & 450811097114200 & 07-20-2006 & 1255 & $<.027$ & $<.006$ & $<.028$ & $<.003$ & $<.007$ & $<.003$ & $<.010$ & $<.015$ & $<.004$ \\
\hline S14 & 452129097111500 & 07-20-2006 & 1135 & $<.027$ & $<.006$ & $<.028$ & $<.003$ & $<.007$ & $<.003$ & $<.010$ & $<.015$ & $<.004$ \\
\hline $\mathrm{S} 15$ & 452214097095900 & 07-20-2006 & 1105 & $<.027$ & $<.006$ & $<.028$ & $<.003$ & $<.007$ & $<.003$ & $<.010$ & $<.015$ & $<.004$ \\
\hline S17 & 452540097142000 & 07-20-2006 & 1040 & $<.027$ & $<.006$ & $<.028$ & $<.003$ & $<.007$ & $<.003$ & $<.010$ & $<.015$ & $<.004$ \\
\hline $\mathrm{S} 18$ & 453354097040800 & 07-13-2006 & 1120 & $<.027$ & E.004 & $<.028$ & $<.003$ & $<.007$ & $<.003$ & $<.010$ & $<.015$ & $<.004$ \\
\hline${ }^{2} \mathrm{~S} 18$ & 453354097040800 & 07-13-2006 & 1130 & $<.027$ & E.004 & $<.028$ & $<.003$ & $<.007$ & $<.003$ & $<.010$ & $<.015$ & $<.004$ \\
\hline S19 & 455145097072900 & 07-11-2006 & 1025 & $<.027$ & $<.006$ & $<.028$ & $<.003$ & $<.007$ & $<.003$ & $<.010$ & $<.015$ & $<.004$ \\
\hline $\mathrm{S} 20$ & 455941097223800 & 07-11-2006 & 1240 & $<.027$ & $<.006$ & $<.028$ & $<.003$ & $<.007$ & $<.003$ & $<.010$ & $<.015$ & $<.004$ \\
\hline $\mathrm{S} 21$ & 454315097184400 & 07-13-2006 & 0850 & $<.027$ & $<.006$ & $<.028$ & $<.003$ & $<.007$ & $<.003$ & $<.010$ & $<.015$ & $<.004$ \\
\hline $\mathrm{S} 22$ & 454012097181700 & 07-13-2006 & 0930 & $<.027$ & $<.006$ & $<.028$ & $<.003$ & $<.007$ & $<.003$ & $<.010$ & $<.015$ & $<.004$ \\
\hline$R P D^{3}$ & & & & 0 & 0 & 0 & 0 & 0 & 0 & 0 & 0 & 0 \\
\hline
\end{tabular}


Table 4. Pesticide concentrations in water samples from selected Lake Traverse Reservation wetlands, July 2006.—Continued

[Location of sites shown in figure 1. All concentrations in micrograms per liter. All concentrations are dissolved, unless otherwise noted. RPD, relative percent difference; AMPA, aminomethylphosphonic acid; EPTC, s-ethyl dipropylcarbomothiate; <, less than; E, estimated. Shading represents estimated values or values greater than the reporting level. Number in parentheses below constituent is U.S. Geological Survey National Water Information System parameter code]

\begin{tabular}{|c|c|c|c|c|c|c|c|c|c|c|c|c|}
\hline Site & Station number & $\begin{array}{c}\text { Date } \\
\text { (mm-dd-yyyy) }\end{array}$ & Time & $\begin{array}{l}\text { Pendi- } \\
\text { methalin } \\
(\mathbf{8 2 6 8 3 )}\end{array}$ & $\begin{array}{c}\text { Phorate } \\
\text { (82664) }\end{array}$ & $\begin{array}{c}\text { Picloram, } \\
\text { total } \\
(39720)\end{array}$ & $\begin{array}{l}\text { Prometon } \\
\text { (04037) }\end{array}$ & $\begin{array}{c}\text { Propyz- } \\
\text { amide } \\
\text { (82676) }\end{array}$ & $\begin{array}{c}\text { Propa- } \\
\text { chlor } \\
\text { (04024) }\end{array}$ & $\begin{array}{c}\text { Propanil } \\
\text { (82679) }\end{array}$ & $\begin{array}{l}\text { Propar- } \\
\text { gite } \\
(82685)\end{array}$ & $\begin{array}{c}\text { Silvex, } \\
\text { total } \\
(39760)\end{array}$ \\
\hline S01 & 454535097023100 & 07-11-2006 & 0910 & $<0.022$ & $<0.055$ & $<0.04$ & $<0.01$ & $<0.004$ & $<0.010$ & $<0.011$ & $<0.02$ & $<0.03$ \\
\hline${ }^{1} \mathrm{~S} 01$ & 454535097023100 & 07-11-2006 & 0900 & $<.022$ & $<.055$ & $<.04$ & $<.01$ & $<.004$ & $<.010$ & $<.011$ & $<.02$ & $<.03$ \\
\hline $\mathrm{S} 02$ & 454717096493000 & $07-12-2006$ & 0930 & $<.022$ & $<.055$ & $<.04$ & $<.01$ & $<.004$ & $<.010$ & $<.011$ & $<.02$ & $<.03$ \\
\hline S03 & 455426096545100 & 07-11-2006 & 1355 & $<.022$ & $<.055$ & $<.04$ & $<.01$ & $<.004$ & $<.010$ & $<.011$ & $<.02$ & $<.03$ \\
\hline S04 & 454933096361200 & 07-12-2006 & 1130 & $<.022$ & $<.055$ & $<.04$ & E.01 & $<.004$ & $<.010$ & $<.011$ & $<.02$ & $<.03$ \\
\hline S07 & 453107096583500 & 07-20-2006 & 0950 & $<.022$ & $<.055$ & $<.04$ & $<.01$ & $<.004$ & $<.010$ & $<.021$ & $<.02$ & $<.03$ \\
\hline S08 & 453031097011800 & 07-20-2006 & 0910 & $<.022$ & $<.055$ & $<.04$ & $<.01$ & $<.004$ & $<.010$ & $<.011$ & $<.02$ & $<.03$ \\
\hline S09 & 453245097071800 & 07-13-2006 & 1030 & $<.022$ & $<.055$ & $<.04$ & $<.01$ & $<.004$ & $<.010$ & $<.011$ & $<.04$ & $<.03$ \\
\hline $\mathrm{S} 10$ & 453801097120100 & 07-12-2006 & 1440 & $<.022$ & $<.055$ & $<.04$ & $<.01$ & $<.004$ & $<.010$ & $<.011$ & $<.02$ & $<.03$ \\
\hline $\mathrm{S} 12$ & 453825097104400 & 07-12-2006 & 1540 & $<.022$ & $<.055$ & $<.04$ & $<.01$ & $<.004$ & $<.010$ & $<.011$ & $<.02$ & $<.03$ \\
\hline S13 & 450811097114200 & 07-20-2006 & 1255 & $<.022$ & $<.055$ & .05 & $<.01$ & $<.004$ & $<.010$ & $<.011$ & $<.04$ & $<.03$ \\
\hline S14 & 452129097111500 & 07-20-2006 & 1135 & $<.022$ & $<.055$ & $<.04$ & $<.01$ & $<.004$ & $<.010$ & $<.011$ & $<.02$ & $<.03$ \\
\hline S15 & 452214097095900 & 07-20-2006 & 1105 & $<.022$ & $<.055$ & $<.04$ & $<.01$ & $<.004$ & $<.010$ & $<.011$ & $<.02$ & $<.03$ \\
\hline $\mathrm{S} 17$ & 452540097142000 & 07-20-2006 & 1040 & $<.022$ & $<.055$ & $<.04$ & $<.01$ & $<.004$ & $<.010$ & $<.015$ & $<.02$ & $<.03$ \\
\hline S18 & 453354097040800 & 07-13-2006 & 1120 & $<.022$ & $<.055$ & $<.04$ & E.01 & $<.004$ & $<.010$ & $<.011$ & $<.02$ & $<.03$ \\
\hline${ }^{2} \mathrm{~S} 18$ & 453354097040800 & 07-13-2006 & 1130 & $<.022$ & $<.055$ & $<.04$ & E.01 & $<.004$ & $<.010$ & $<.011$ & $<.02$ & $<.03$ \\
\hline S19 & 455145097072900 & 07-11-2006 & 1025 & $<.022$ & $<.055$ & $<.04$ & .03 & $<.004$ & $<.010$ & $<.011$ & $<.02$ & $<.03$ \\
\hline S20 & 455941097223800 & 07-11-2006 & 1240 & $<.022$ & $<.055$ & $<.04$ & $<.01$ & $<.004$ & $<.010$ & $<.011$ & $<.02$ & $<.03$ \\
\hline S21 & 454315097184400 & 07-13-2006 & 0850 & $<.022$ & $<.055$ & $<.04$ & $<.01$ & $<.004$ & $<.010$ & $<.011$ & $<.02$ & $<.03$ \\
\hline $\mathrm{S} 22$ & 454012097181700 & 07-13-2006 & 0930 & $<.022$ & $<.055$ & $<.04$ & $<.01$ & $<.004$ & $<.010$ & $<.011$ & $<.04$ & $<.03$ \\
\hline$R P D^{3}$ & & & & 0 & 0 & 0 & 0 & 0 & 0 & 0 & 0 & 0 \\
\hline
\end{tabular}


[Location of sites shown in figure 1. All concentrations in micrograms per liter. All concentrations are dissolved, unless otherwise noted. RPD, relative percent difference; AMPA, aminomethylphosphonic acid; EPTC, s-ethyl dipropylcarbomothiate; <, less than; E, estimated. Shading represents estimated values or values greater than the reporting level. Number in parentheses below constituent is U.S. Geological Survey National Water Information System parameter code]

\begin{tabular}{|c|c|c|c|c|c|c|c|c|c|c|}
\hline Site & Station number & $\begin{array}{c}\text { Date } \\
\text { (mm-dd-yyyy) }\end{array}$ & Time & $\begin{array}{c}\text { Simazine } \\
(04035)\end{array}$ & $\begin{array}{c}\text { Tebuthi- } \\
\text { uron } \\
(\mathbf{8 2 6 7 0 )}\end{array}$ & $\begin{array}{l}\text { Terbacil } \\
\text { (82665) }\end{array}$ & $\begin{array}{c}\text { Terbufos } \\
\text { (82675) }\end{array}$ & $\begin{array}{c}\text { Thioben- } \\
\text { carb } \\
\text { (82681) }\end{array}$ & $\begin{array}{c}\text { Triallate } \\
\text { (82678) }\end{array}$ & $\begin{array}{c}\text { Trifluralin } \\
\text { (82661) }\end{array}$ \\
\hline S01 & 454535097023100 & 07-11-2006 & 0910 & $<0.005$ & $<0.02$ & $<0.034$ & $<0.02$ & $<0.010$ & $<0.006$ & $<0.009$ \\
\hline${ }^{1} \mathrm{~S} 01$ & 454535097023100 & 07-11-2006 & 0900 & $<.005$ & $<.02$ & $<.034$ & $<.02$ & $<.010$ & $<.006$ & $<.009$ \\
\hline S02 & 454717096493000 & 07-12-2006 & 0930 & $<.005$ & $<.02$ & $<.034$ & $<.02$ & $<.010$ & $<.006$ & $<.009$ \\
\hline S03 & 455426096545100 & 07-11-2006 & 1355 & $<.005$ & $<.02$ & $<.034$ & $<.02$ & $<.010$ & $<.006$ & $<.009$ \\
\hline S04 & 454933096361200 & 07-12-2006 & 1130 & E.002 & $<.02$ & $<.034$ & $<.02$ & $<.010$ & $<.006$ & $<.009$ \\
\hline S07 & 453107096583500 & 07-20-2006 & 0950 & $<.005$ & $<.02$ & $<.034$ & $<.02$ & $<.010$ & $<.006$ & $<.009$ \\
\hline S08 & 453031097011800 & 07-20-2006 & 0910 & $<.005$ & $<.02$ & $<.034$ & $<.02$ & $<.010$ & $<.006$ & $<.009$ \\
\hline S09 & 453245097071800 & 07-13-2006 & 1030 & $<.005$ & $<.02$ & $<.034$ & $<.02$ & $<.010$ & $<.006$ & $<.009$ \\
\hline $\mathrm{S} 10$ & 453801097120100 & 07-12-2006 & 1440 & $<.005$ & $<.02$ & $<.034$ & $<.02$ & $<.010$ & $<.006$ & $<.009$ \\
\hline $\mathrm{S} 12$ & 453825097104400 & 07-12-2006 & 1540 & $<.005$ & $<.02$ & $<.034$ & $<.02$ & $<.010$ & $<.006$ & $<.009$ \\
\hline $\mathrm{S} 13$ & 450811097114200 & 07-20-2006 & 1255 & $<.005$ & $<.02$ & $<.034$ & $<.02$ & $<.010$ & $<.006$ & $<.009$ \\
\hline $\mathrm{S} 14$ & 452129097111500 & 07-20-2006 & 1135 & $<.005$ & $<.02$ & $<.034$ & $<.02$ & $<.010$ & $<.006$ & $<.009$ \\
\hline $\mathrm{S} 15$ & 452214097095900 & 07-20-2006 & 1105 & $<.005$ & $<.02$ & $<.034$ & $<.02$ & $<.010$ & $<.006$ & $<.009$ \\
\hline $\mathrm{S} 17$ & 452540097142000 & 07-20-2006 & 1040 & $<.005$ & $<.02$ & $<.034$ & $<.02$ & $<.010$ & $<.006$ & $<.009$ \\
\hline $\mathrm{S} 18$ & 453354097040800 & 07-13-2006 & 1120 & $<.005$ & $<.02$ & $<.034$ & $<.02$ & $<.010$ & $<.006$ & $<.009$ \\
\hline${ }^{2} \mathrm{~S} 18$ & 453354097040800 & 07-13-2006 & 1130 & $<.005$ & $<.02$ & $<.034$ & $<.02$ & $<.010$ & $<.006$ & $<.009$ \\
\hline S19 & 455145097072900 & 07-11-2006 & 1025 & $<.005$ & $<.02$ & $<.034$ & $<.02$ & $<.010$ & $<.006$ & $<.009$ \\
\hline $\mathrm{S} 20$ & 455941097223800 & 07-11-2006 & 1240 & $<.005$ & $<.02$ & $<.034$ & $<.02$ & $<.010$ & $<.006$ & $<.009$ \\
\hline $\mathrm{S} 21$ & 454315097184400 & 07-13-2006 & 0850 & $<.005$ & $<.02$ & $<.034$ & $<.02$ & $<.010$ & $<.006$ & $<.009$ \\
\hline $\mathrm{S} 22$ & 454012097181700 & 07-13-2006 & 0930 & $<.005$ & $<.02$ & $<.034$ & $<.02$ & $<.010$ & $<.006$ & $<.009$ \\
\hline$R P D^{3}$ & & & & 0 & 0 & 0 & 0 & 0 & 0 & 0 \\
\hline
\end{tabular}

${ }^{1}$ Field equipment blank sample collected at site (water only).

${ }^{2}$ Replicate sample collected at site.

${ }^{3}$ Relative percent difference between field environmental and field replicate sample for site S18. 


\section{Pesticide Concentrations in Wetlands}

This section of the report summarizes the pesticide concentrations in water samples collected from 18 wetlands within the Lake Traverse Reservation. In addition to presenting analytical results for pesticides, results for physical properties are included. The samples collected for this study were analyzed for the physical properties of barometric pressure, turbidity, dissolved oxygen, $\mathrm{pH}$, specific conductance, water temperature, and air temperature (table 3), and 61 pesticides (table 4).

\section{Physical Properties}

Water samples collected for this study were analyzed for the physical properties presented in table 3 . The results indicate the wetlands are representative of a wide variety of conditions. Turbidity ranged from 0.5 formazin nephelometric turbidity unit (FNU) (wetland site S17) to $230 \mathrm{FNU}$ (wetland site S12), with a median value of 6.1 FNU. Dissolved-oxygen concentrations ranged from 1.4 milligrams per liter $(\mathrm{mg} / \mathrm{L})$ (wetland site S19) to $18.5 \mathrm{mg} / \mathrm{L}$ (wetland site S12), with a median concentration of $6 \mathrm{mg} / \mathrm{L}$. The $\mathrm{pH}$ values ranged from 6.7 (wetland site S13) to 9.4 (wetland site S17), with a median value of 8.2. Specific conductance ranged from 272 microsiemens per centimeter $(\mu \mathrm{S} / \mathrm{cm})$ (wetland site S22) to $5,040 \mu \mathrm{S} / \mathrm{cm}$ (wetland site S07), with a median value of $891 \mu \mathrm{S} / \mathrm{cm}$. Water temperature ranged from 18.1 degrees Celsius $\left({ }^{\circ} \mathrm{C}\right.$ ) (wetland site S14) to $32.7^{\circ} \mathrm{C}$ (wetland site S12), with a median temperature of $23.5^{\circ} \mathrm{C}$.

\section{Pesticides}

Water samples were analyzed for dissolved concentrations of 55 pesticides and for total concentrations of 6 pesticides (table 4). Of the 61 pesticides analyzed for in samples, 51 were not detected in any of the samples. Of the 10 pesticides that had estimated concentrations or concentrations greater than the LRL (fig. 2), most were detected at very low concentrations in only a few samples.

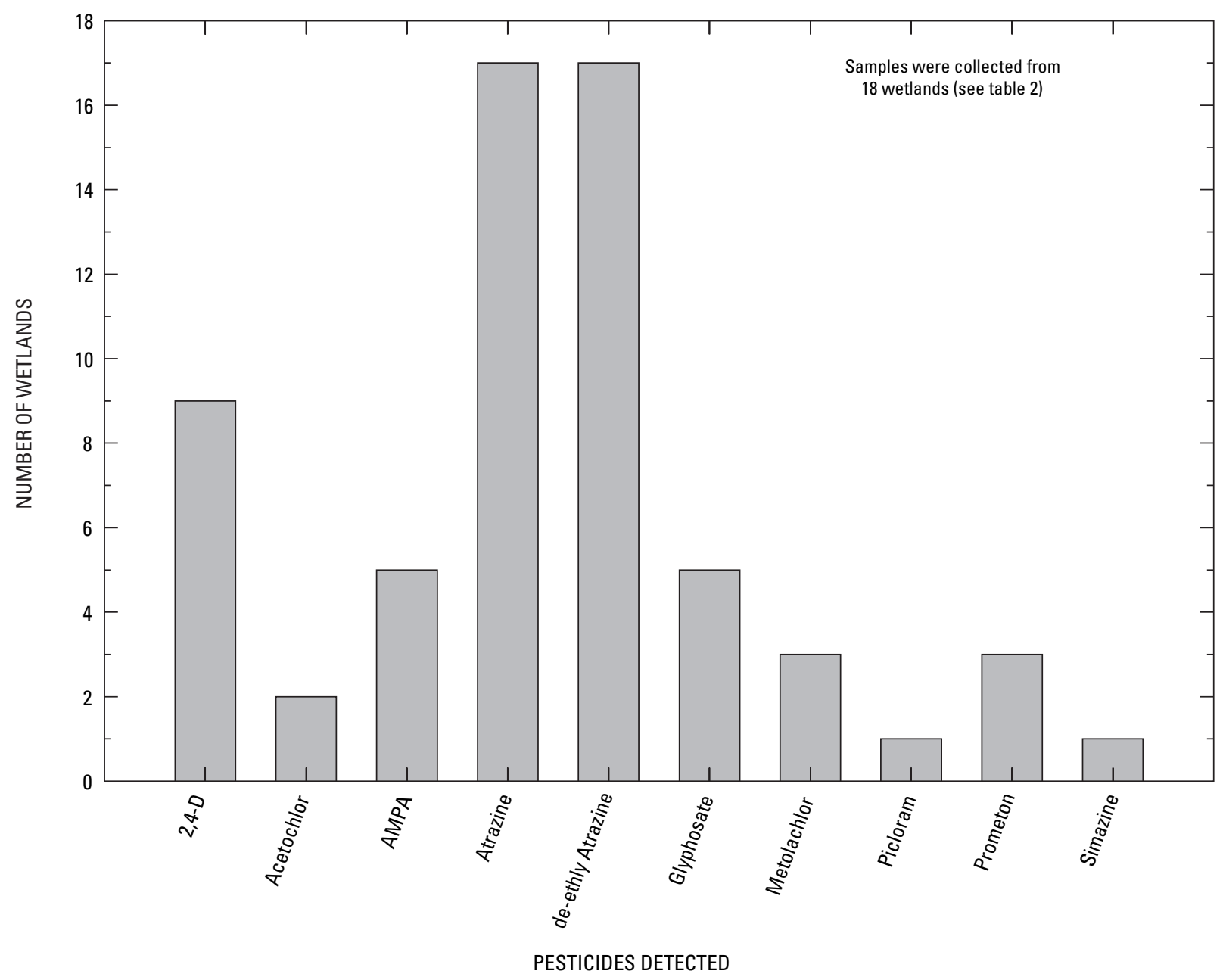

Figure 2. Number of Lake Traverse Reservation wetlands at which selected pesticides were detected, July 2006. 
Samples from all but one wetland (site S14) contained at least two pesticides (fig. 3); no pesticides were detected at site 14 . Of the 17 wetlands in which pesticides were detected, the number of pesticides detected ranged from two to eight, with a median of four.

Atrazine and the atrazine metabolite, de-ethyl atrazine, were detected in 17 of the 18 wetlands sampled (fig. 2). Detected concentrations of dissolved atrazine ranged from 0.007 to 0.12 microgram per liter $(\mu \mathrm{g} / \mathrm{L})$, with a median concentration of $0.064 \mu \mathrm{g} / \mathrm{L}$. Estimated concentrations of dissolved de-ethyl atrazine ranged from 0.005 to $0.03 \mu \mathrm{g} / \mathrm{L}$, with a median concentration of $0.017 \mu \mathrm{g} / \mathrm{L}$. Other pesticides that had concentrations that were estimated or greater than the LRL included 2,4-D, which was detected in nine environmental samples and the one replicate sample, with dissolved concentrations that ranged from 0.03 to $0.11 \mu \mathrm{g} / \mathrm{L}$; glyphosate, which was detected in five samples with dissolved concentrations that ranged from an estimated 0.050 to $0.3 \mu \mathrm{g} / \mathrm{L}$; aminomethylphosphonic acid (AMPA), which was detected in five samples with dissolved concentrations that ranged from an estimated 0.090 to $0.46 \mu \mathrm{g} / \mathrm{L}$; metolachlor, which was detected in three environmental samples and the one replicate sample, with dissolved concentrations that ranged from an estimated 0.004 to $0.027 \mu \mathrm{g} / \mathrm{L}$; prometon, which was detected in three environmental samples and the one replicate sample, with dissolved concentrations that ranged from an estimated 0.01 to $0.03 \mu \mathrm{g} / \mathrm{L}$; acetochlor, which was detected in two environmental samples and the one replicate sample, with estimated dissolved concentrations that ranged from 0.007 to $0.009 \mu \mathrm{g} / \mathrm{L}$; picloram, which was detected in one sample at a concentration of $0.05 \mu \mathrm{g} / \mathrm{L}$; and simazine, which was detected in one sample at a dissolved concentration of an estimated $0.002 \mu \mathrm{g} / \mathrm{L}$.

\section{Synopsis of Pesticide Results}

The analytical results from this study indicate some pesticides were present in selected Lake Traverse Reservation wetlands. Comparing these results to those from previous studies and to established water-quality standards may provide useful information relative to the quality of the water in the wetlands. This synopsis will focus on the pesticides detected at concentrations greater than the LRLs for this study.

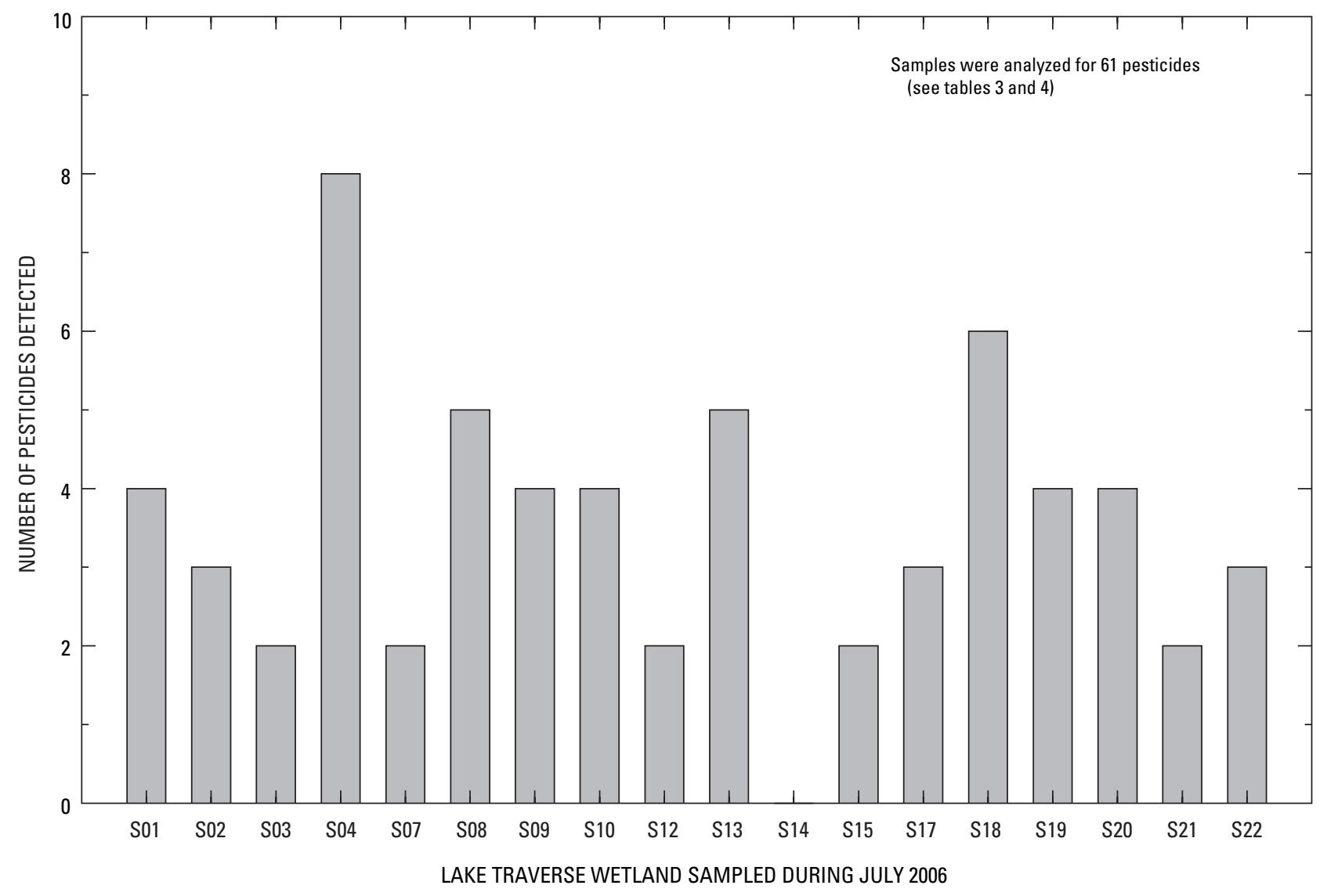

Figure 3. Number of pesticides detected in water samples for selected Lake Traverse Reservation wetlands, July 2006. 
The U.S. Environmental Protection Agency (USEPA) has established drinking water standards for some compounds that may cause adverse health effects from long-term exposure. These standards are known as Maximum Contaminant Levels (MCLs) (U.S. Environmental Protection Agency, 2002).

Because MCLs apply only to drinking water from public water supplies, the MCLs do not apply to any of the wetlands in this study, but are used as a basis of comparison.

\section{Atrazine}

Atrazine has been widely used in the study area (table 1) and has the physical and chemical properties that allow it to move readily in the environment (Capel, 1990). Atrazine was one of 34 pesticides for which Battaglin and Goolsby (1994) estimated use in Roberts County in 1987. Of the 34 pesticides, atrazine was ranked ninth in the number of acres treated and eighth in pounds of active ingredient applied (table 1).

Atrazine (along with its metabolite, de-ethyl atrazine) was detected more frequently (fig. 2) than any other pesticide in wetlands (17 of 18 wetlands) selected for this study and had detected concentrations that ranged from 0.007 to $0.12 \mu \mathrm{g} / \mathrm{L}$. Atrazine also was commonly detected in two samples from surface-water sites in the study area (Big Sioux River and Indian River) sampled by the SDGS in 1993 at concentrations that ranged from 0.1 to $1.5 \mu \mathrm{g} / \mathrm{L}$ (Thompson, 2002). The SDGS also sampled nine observation wells in the study area and found atrazine concentrations that ranged from 0.1 to $0.7 \mu \mathrm{g} / \mathrm{L}$ (Thompson, 2002). Atrazine also was detected in samples collected during June 2004 from all nine Big Sioux River sites near Watertown, Volga, and Brookings at concentrations that ranged from an estimated 0.16 to $1.5 \mu \mathrm{g} / \mathrm{L}$, but was not as frequently detected in samples collected at the same sites during August 2003 (Sando and others, 2005). Atrazine was detected in all 18 wetland samples collected in 2005 from the Yankton Sioux Reservation at concentrations that ranged from 0.056 to $0.151 \mu \mathrm{g} / \mathrm{L}$ (Schaap and Bartholomay, 2006).

For this study and previous studies, samples analyzed for atrazine were collected during periods when the concentrations probably were at their largest concentrations because of recent application to fields. Although the detection frequency was high, concentrations for this study were much less than the MCL for drinking water of $3 \mu \mathrm{g} / \mathrm{L}$ (U.S. Environmental Protection Agency, 2002). Field and laboratory studies have indicated that atrazine may be associated with endocrine disruption in aquatic organisms (Hayes and others, 2003, 2006; Spano and others, 2004). Atrazine exposure has been reported to result in reproductive abnormalities in American Leopard frogs at concentrations as small as $0.1 \mu \mathrm{g} / \mathrm{L}$ (Hayes and others, 2003), but reported levels for fish are substantially larger (Sando and others, 2005). The atrazine concentrations detected in two samples from this study were at the levels that may affect reproduction in frogs, but the length of time the concentrations remained at this level in the environment cannot be determined without more sampling. Further research is needed to fully assess the potential for endocrine disruption.

\section{2,4-D}

2,4-D was one of the most widely used pesticides in the study area (table 1) and the second most frequently detected compound (fig. 2). 2,4-D was detected in 9 of 18 wetlands sampled. It was one of 34 pesticides for which Battaglin and Goolsby (1994) estimated use in Roberts County in 1987. Of the 34 pesticides, 2,4-D ranked first in number of acres treated and fifth in pounds of active ingredient applied (table 1).

2,4-D concentrations in Lake Traverse Reservation wetlands ranged from 0.03 to $0.11 \mu \mathrm{g} / \mathrm{L}$. 2,4-D also was detected in three samples from two surface-water sites in the study area (Big Sioux River and Indian River) sampled by SDGS in 1993 at concentrations that ranged from 1.0 to $7.5 \mu \mathrm{g} / \mathrm{L}$ (Thompson, 2002). The SDGS also sampled nine observation wells in the study area and found 2,4-D concentrations that ranged from 0.1 to $0.7 \mu \mathrm{g} / \mathrm{L}$ (Thompson, 2002). 2,4-D also was detected in 12 wetland samples collected in 2005 from the Yankton Sioux Reservation at concentrations that ranged from an estimated 0.02 to $0.85 \mu \mathrm{g} / \mathrm{L}$ (Schaap and Bartholomay, 2006).

2,4-D samples for this study and other previous studies were collected during periods when the concentrations were probably at their largest concentrations because of recent application to fields. Although the detection frequency was high, concentrations for this study were much less than the MCL for drinking water of $70 \mu \mathrm{g} / \mathrm{L}$ (U.S. Environmental Protection Agency, 2002). Field and laboratory studies have indicated that 2,4-D and its forms range from being practically nontoxic to highly toxic to fish and aquatic invertebrates (U.S. Forest Service, 1995), but toxicity occurs at concentrations that are much larger than those detected in this study.

\section{Glyphosate}

Glyphosate was a commonly used pesticide in the study area (table 1) and was the third most frequently detected compound (fig. 2), along with its primary transformation product AMPA. Glyphosate was detected in 5 of the 18 wetlands sampled (fig. 2). It was one of 34 pesticides for which Battaglin and Goolsby (1994) estimated use in Roberts County in 1987. Of the 34 pesticides, glyphosate was ranked twelfth in number of acres treated and fourteenth in pounds of active ingredient applied (table 1); however, its use in the midwestern United States tripled from 1997 to 2001 (Battaglin and others, 2003).

Glyphosate concentrations ranged from an estimated 0.050 to $0.3 \mu \mathrm{g} / \mathrm{L}$. Glyphosate was not detected in previous samples from the study area (Thompson, 2002), but was detected in eight wetland samples collected in 2005 from the Yankton Sioux Reservation at concentrations that ranged from 0.1 to $0.7 \mu \mathrm{g} / \mathrm{L}$ (Schaap and Bartholomay, 2006). 
Glyphosate samples for this study and other previous studies were collected during periods when the concentrations probably were at their largest concentrations because of recent application to fields. Concentrations for this study were much less than the MCL for drinking water of $700 \mu \mathrm{g} / \mathrm{L}$ (U.S. Environmental Protection Agency, 2002). Field and laboratory studies have indicated that glyphosate is only slightly toxic to birds, fish, and aquatic invertebrates and does not bioaccumulate (Battaglin and others, 2003). Toxicity to aquatic organisms occurs at concentrations that are orders of magnitude larger than what was detected in this study.

\section{Metolachlor}

Metolachlor was a commonly used pesticide in the study area (table 1) and was detected in 3 of the 18 wetlands sampled (fig. 2). Metolachlor was one of 34 pesticides for which Battaglin and Goolsby (1994) estimated use in Roberts County in 1987. Of the 34 pesticides, metolachlor was ranked eighth in number of acres treated and fourth in pounds of active ingredient applied (table 1); however, its use in the midwestern United States decreased by 55 percent from 1997 to 2001 (Battaglin and others, 2003).

Metolachlor concentrations ranged from an estimated 0.004 to $0.027 \mu \mathrm{g} / \mathrm{L}$. Metolachlor was detected in two previous samples from the study area (Thompson, 2002) and was detected in 17 wetland samples collected in 2005 from the Yankton Sioux Reservation at concentrations that ranged from 0.006 to $1.89 \mu \mathrm{g} / \mathrm{L}$ (Schaap and Bartholomay, 2006).

Metolachlor samples for this study and other previous studies were collected during periods when the concentrations probably were at their largest concentrations because of recent application to fields. No MCL for drinking water has been established for metolachlor. Field and laboratory studies have indicated that metolachlor is slightly toxic to some aquatic species (Battaglin and Fairchild, 2002), but toxicity occurs at concentrations that are orders of magnitude larger than what was detected in this study.

\section{Prometon}

Prometon was detected in three wetlands in the study area (fig. 2), but was not one of 34 pesticides for which Battaglin and Goolsby (1994) estimated use in Roberts County in 1987. Prometon concentrations ranged from an estimated 0.01 to $0.03 \mu \mathrm{g} / \mathrm{L}$. Prometon was not detected in previous samples from the study area (Thompson, 2002) nor in samples collected in 2005 from the Yankton Sioux Reservation (Schaap and Bartholomay, 2006).

Prometon samples for this study and other previous studies were collected during periods when the concentrations probably were at their largest concentrations because of recent application to fields. No MCL for drinking water has been established for prometon. Field and laboratory studies have indicated that prometon is slightly toxic to green algae and bluegill sunfish (Battaglin and Fairchild, 2002), but toxicity occurs at concentrations that are orders of magnitude larger than what was detected in this study.

\section{Acetochlor}

Acetochlor was detected in two wetlands in the study area (fig. 2), but was not one of 34 pesticides for which Battaglin and Goolsby (1994) estimated use in Roberts County in 1987. Battaglin and others (2003) estimated the use of acetochlor had increased in the midwestern United States by about 10 percent from 1997 to 2001 . Acetochlor, which was detected in two environmental samples and the one replicate sample, had estimated dissolved concentrations that ranged from 0.007 to $0.009 \mu \mathrm{g} / \mathrm{L}$. Acetochlor was not detected in previous samples from the study area (Thompson, 2002) but was detected in nine wetland samples collected in 2005 from the Yankton Sioux Reservation at concentrations that ranged from 0.011 to $0.039 \mu \mathrm{g} / \mathrm{L}$ (Schaap and Bartholomay, 2006).

Acetochlor samples for this study and other previous studies were collected during periods when the concentrations probably were at their largest concentrations because of recent application to fields. No MCL for drinking water has been established for acetochlor. Field and laboratory studies have indicated that acetochlor is slightly toxic to some aquatic species (Battaglin and Fairchild, 2002), but toxicity occurs at concentrations that are orders of magnitude larger than what was detected in this study.

\section{Picloram}

Picloram was detected in one wetland in the study area (fig. 2), and was one of 34 pesticides for which Battaglin and Goolsby (1994) estimated use in Roberts County in 1987. The picloram concentration was $0.05 \mu \mathrm{g} / \mathrm{L}$. Picloram was not detected in previous samples from the study area (Thompson, 2002) but was detected in nine wetland samples collected in 2005 from the Yankton Sioux Reservation at concentrations that ranged from an estimated 0.01 to $0.88 \mu \mathrm{g} / \mathrm{L}$ (Schaap and Bartholomay, 2006).

Picloram samples for this study and other previous studies were collected during periods when the concentrations probably were at their largest concentrations because of application to fields. The MCL for picloram in drinking water is $500 \mu \mathrm{g} / \mathrm{L}$ (U.S. Environmental Protection Agency, 2002). Field and laboratory studies have indicated that picloram is slightly to moderately toxic to some birds, fish, and other aquatic species, but toxicity occurs at concentrations that are orders of magnitude larger than what was detected in this study (EXTOXNET, 2006). 


\section{Summary}

During July 2006, water samples were collected from selected Lake Traverse Reservation wetlands within the historic Reservation boundary in northeastern South Dakota and southeastern North Dakota as part of a reconnaissancelevel assessment by the U.S. Geological Survey (USGS) and Sisseton-Wahpeton Oyate (SWO). Eighteen wetlands were sampled by the SWO and USGS personnel after primary pesticide application occurred on the Lake Traverse Reservation. Samples were analyzed for 61 pesticides and for physical properties that might help further characterize the water quality of the wetlands.

Analysis results of the physical properties indicate that a wide variety of conditions occur among the wetlands. Turbidity ranged from 0.5 to 230 formazin nephelometric turbidity units. Dissolved-oxygen concentrations ranged from 1.4 to 18.5 milligrams per liter. The $\mathrm{pH}$ values ranged from 6.7 to 9.4. Specific conductance ranged from 272 to 5,040 microsiemens per centimeter. Water temperature ranged from 18.1 to 32.7 degrees Celsius.

Pesticides were detected in 17 of the 18 sampled wetlands. In the 17 wetlands in which pesticides were detected, the number of pesticides detected ranged from two to eight, with a median of four. Of the 61 pesticides for which the samples were analyzed, 51 pesticides were not detected in any of the wetland samples. The 10 detected pesticides had small concentrations. Atrazine and de-ethyl atrazine were detected in samples from 17 of the 18 wetlands. Concentrations of dissolved atrazine ranged from 0.007 to 0.12 microgram per liter $(\mu \mathrm{g} / \mathrm{L})$. Estimated concentrations of dissolved de-ethyl atrazine ranged from 0.005 to $0.03 \mu \mathrm{g} / \mathrm{L}$. Other pesticides that had concentrations that were estimated or greater than the laboratory reporting level included 2,4-D, which was detected in nine samples with dissolved concentrations that ranged from 0.03 to $0.11 \mu \mathrm{g} / \mathrm{L}$; glyphosate, which was detected in five samples with dissolved concentrations that ranged from an estimated 0.050 to $0.3 \mu \mathrm{g} / \mathrm{L}$; aminomethylphosphonic acid (AMPA), which was detected in five samples with dissolved concentrations that ranged from an estimated 0.090 to $0.46 \mu \mathrm{g} / \mathrm{L}$; metolachlor, which was detected in three samples with dissolved concentrations that ranged from an estimated 0.004 to $0.027 \mu \mathrm{g} / \mathrm{L}$; prometon, which was detected in three samples with dissolved concentrations that ranged from an estimated 0.01 to $0.03 \mu \mathrm{g} / \mathrm{L}$; acetochlor, which was detected in two environmental samples and the one replicate sample, with estimated dissolved concentrations that ranged from 0.007 to $0.009 \mu \mathrm{g} / \mathrm{L}$; picloram, which was detected in one sample at a concentration of $0.05 \mu \mathrm{g} / \mathrm{L}$; and simazine, which was detected in one sample at a dissolved concentration of an estimated $0.002 \mu \mathrm{g} / \mathrm{L}$.

None of the pesticides detected were greater than any U.S. Environmental Protection Agency Maximum Contaminant Levels for drinking water. Concentrations were much less than levels considered toxic for humans or aquatic species.

\section{References Cited}

Amundson, F.D., 2002, Estimated use of water in South Dakota: U.S. Geological Survey Open-File Report 02-440, 17 p., accessed January 4, 2007, at http://pubs.water.usgs. gov/ofr02440

Battaglin, W.A., and Fairchild, J.F., 2002, Potential toxicity of pesticides measured in midwestern streams to aquatic organisms: Water Science and Technology, v. 45, no. 9, p. 95-103, accessed January 4, 2007, at http://co.water.usgs. gov/midconherb/pdf/wst_battagl.pdf

Battaglin, W.A., and Goolsby, D.A., 1994, Spatial data in geographic information system format on agricultural chemical use, land use, and cropping practices in the United States: U.S. Geological Survey Water-Resources Investigations Report 94-4176, 87 p., accessed January 4, 2007, at http://pubs.usgs.gov/wri/wri944176/.

Battaglin, W.A., Thurman, E.M., Kolpin, D.W., Scribner, E.A., Sandstrom, M.W., and Kuivila, K.M., 2003, Work plan for determining the occurrence of glyphosate, its transformation product AMPA, other herbicide compounds, and antibiotics in Midwestern United States streams, 2002: U.S. Geological Survey Open-File Report 03-69, 18 p., accessed January 4, 2007, at http://pubs.usgs.gov/of/2003/ofr03-069/.

Capel, P.D., 1990, A bibliography of atrazine and its simple degradation products in the environment: U.S. Geological Survey Open-File Report 89-613, 5 p.

Childress, C.J.O., Foreman, W.T., Connor, B.F., and Maloney, T.J., 1999, New reporting procedures based on longterm method detection levels and some considerations for interpretations of water-quality data provided by the U.S. Geological Survey National Water Quality Laboratory: U.S. Geological Survey Open-File Report 99-193, 19 p.

Danzo, B.J., 1997, Environmental xenobiotics may disrupt normal endocrine function by interfering with the binding of physiological ligands to steroid receptors and binding proteins: Environmental Health Perspectives, v. 105, p. 294-301.

EXTOXNET, 2006, Pesticide information profiles-Picloram: accessed December 13, 2006, at http://extoxnet.orst.edu/ pips/picloram.htm

Gilliom, R.J., Barbash, J.E., Crawford, C.G., Hamilton, P.A., Martin, J.D., Nakagaki, Naomi, Nowell, L.H., Scott, J.C., Stackelberg, P.E., Thelin, G.P., and Wolock, D.M., 2006, The quality of our Nation's waters-Pesticides in the Nation's streams and ground water, 1992-2001: U.S. Geological Survey Circular 1291, 184 p., accessed January 4, 2007, at http://pubs.usgs.gov/circ/2005/1291/. 
Hayes, T.B.; Case, Paola; Chui, Sarah; Chung, Duc; Haeffele, Cathryn; Haston, Kelly; Lee, Melissa; Phoung Mai, Vien; Marjuoa, Youssra; Parker, John; and Tsui, Mable, 2006, Pesticide mixtures, endocrine disruption, and amphibian declines: Are we underestimating the impact?: Environmental Health Perspectives, v. 114, no. S-1, p. $40-50$.

Hayes, Tyrone; Haston, Kelly; Tsui, Mable; Hoang, Anhthu; Haeffle, Cathryn; and Vonk, Aaron, 2003, Atrazineinduced hermaphroditism at $0.1 \mathrm{ppb}$ in American leopard frogs (Rana pipiens) - Laboratory and field evidence: Environmental Health Perspectives, v. 111, no. 4, p. 568-575.

Lawrence, S.J., 1989, Water-resources appraisal of the Lake Traverse Indian Reservation County in South Dakota: U.S. Geological Survey Water-Resources Investigations Report 88-4031, 42 p.

Lee, E.A., Straham, A.P., and Thurman, E.M., 2001, Methods of analysis by the U.S. Geological Survey Organic Geochemistry Research Group-Determination of glyphosate, aminomethylphosphonic acid, and glufosinate in water using online solid-phase extraction and highperformance liquid chromatography/mass spectrometry: U.S. Geological Survey Open-File Report 01-454, 13 p., accessed January 4, 2007, at http://ks.water.usgs.gov/ Kansas/pubs/reports/ofr.01-454.html

National Weather Service, 2006a, National Weather Service Forecast Office: accessed November 27, 2006, at http://www.weather.gov/climate/index.php? $w f o=a b r$

National Weather Service, 2006b, Precipitation analysis: accessed November 27, 2006, at http://www.srh.noaa.gov/ rfcshare/precip_analysis_new.php

Rawlings, N.C., Cook, S.J., and Waldbillig, D., 1998, Effects of the pesticides carbofuran, chlorpyrifos, dimethoate, lindane, triallate, trifluralin, 2,4-D, and pentachlorophenol on the metabolic endocrine and reproductive endocrine system in ewes: Journal of Toxicology and Environmental Health, v. 54, p. 21-36.

Sando, S.K., Furlong, E.T., Gray, J.L., Meyer, M.T., and Bartholomay, R.C., 2005, Occurrence of organic wastewater compounds in wastewater effluent and the Big Sioux River in the Upper Big Sioux River Basin, South Dakota, 2003-2004: U.S. Geological Survey Scientific Investigations Report 2005-5249, 108 p., accessed January 4, 2007, at http://pubs.usgs.gov/sir/2005/5249/.
Schaap, B.D., and Bartholomay, R.C., 2006, Reconnaissancelevel assessment of water and bottom-sediment quality, including pesticides and mercury, in Yankton Sioux Tribe wetlands, Charles Mix County, South Dakota, June-July 2005: U.S. Geological Survey Scientific Investigations Report 2006-5132, 40 p., accessed January 4, 2007, at http://pubs.usgs.gov/sir/2006/5132/.

South Dakota Agricultural Statistics Service, 2001, South Dakota 2000 agricultural chemical usage: U.S. Department of Agriculture, accessed July 30, 2001, at http://www.nass. usda.gov/sd/releases/agchem01.pdf

Spano, Laura; Tyler, C.R.; van Aerle, Ronny; Devos, Pierre; Mandiki, S.N.M.; Silvestre, Fredric; Thome, J.P., and Kestemont, Patrick, 2004, Effects of atrazine on sex steroid dynamics, plasma vitellogenin concentration and gonad development in adult goldfish (Carassius auratus): Aquatic Toxicology, v. 66, p. 369-379.

Taylor, J.K., 1987, Quality assurance of chemical measurements: Chelsea, Mich., Lewis Publishers, 328 p.

Thompson, R.F., 2000, Selected hydrologic and water-quality data, 1997 through 1999, for the Lake Traverse Reservation/ Roberts County water-resources investigation in South Dakota: U.S. Geological Survey Open-File Report 00-445, 304 p.

Thompson, R.F., 2001, Water resources of the Lake Traverse Reservation, South and North Dakota, and Roberts County, South Dakota: U.S. Geological Survey Water-Resources Investigations Report 01-4219, 105 p., accessed January 4, 2007, at http://pubs.usgs.gov/wri/wri01-4219/.

Thompson, R.F., 2002, Compilation of data to support development of a pesticide management plan by the Sisseton-Wahpeton Sioux Tribe, South and North Dakota: U.S. Geological Survey Open-File Report 02-381, 25 p.

U.S. Census Bureau, 2001, Population for South Dakota and counties, 1990 and 2000: accessed November 24, 2006, at http://www.usd.edu.brbinfo/.

U.S. Environmental Protection Agency, 2002, List of contaminants \& their Maximum Contaminant Levels (MCLs): accessed November 24, 2006, at http://www.epa.gov/safewater/mcl.html

U.S. Fish and Wildlife Service, 2004, Wetlands information: accessed November 28, 2006, at http://wetlandsfws.er.usgs. gov 
U.S. Forest Service, 1995, 2,4-D pesticide fact sheet: Technical Report of the U.S. Forest Service, accessed March 19, 2004, at http://infoventures.com/e-hlth/pestcide/ 24d.html

U.S. Forest Service, 1997, Glyphosate herbicide information profile: Technical Report of the Pacific Northwest Region, U.S. Forest Service, 16 p., accessed March 19, 2004, at http://www.fs.fed.us/r6/nr/fid/pubsweb/gly.pdf

Wershaw, R.L., Fishman, M.J., Grabbe, R.R., and Lowe, L.E., 1987, Methods for the determination of organic substances in water and fluvial sediments: U.S. Geological Survey Techniques of Water-Resources Investigations, book 5, chap. A3, 80 p.

Wilde, F.D., Radtke, D.B., Gibs, Jacob, and Iwatsubo, R.T., eds., 1999, Collection of water samples: U.S. Geological Survey Techniques of Water-Resources Investigations, book 9, chap. A4, accessed November 29, 2006, at http://pubs. water.usgs.gov/twri9A

Wilson, A.G., Thake, D.C., Heydens, W.C., Brewster, D.W., and Hotz, K.J., 1996, Mode of action of thyroid tumor formation in the male Long-Evans rat administered high doses of alachlor: Fundamental and Applied Toxicology, v. 33 , p. 16-23.

Zaugg, S.D., Sandstrom, M.W., Smith, S.G., and Fehlberg, K.M., 1995, Methods of analysis by the U.S. Geological Survey National Water Quality Laboratory-Determination of pesticides in water by $\mathrm{C}-18$ solid-phase extraction and capillary-column gas chromatography/mass spectrometry with selected-ion monitoring: U.S. Geological Survey Open-File Report 95-181, 60 p. 


\section{Supplemental Information}

\section{U.S. Fish and Wildlife Service Wetlands Inventory Codes and Definitions}

This Supplemental Information section provides information about U.S. Fish and Wildlife Service wetlands inventory codes and definitions for the selected Lake Traverse wetlands at which water samples were collected in July 2006 (table 5). The U.S. Fish and Wildlife Service wetlands inventory for South Dakota was last accessed November 28, 2006, at http://wetlandsfws. er.usgs.gov/. The inventory is in the form of a geographic information system shapefile for individual U.S. Geological Survey quadrangles consisting of delineated wetlands and attributes of the wetlands. One of the attributes for each wetland is a code that indicates how the wetland had been classified in terms of various factors including geomorphology, water quality, vegetation, and the duration of the presence of standing water.

Of the 18 wetland sites at which water samples were collected, two (sites S15 and S18) did not have an associated primary wetland inventory code. Three sites, S02, S10, and S17, have been assigned to the lacustrine system; and the remaining 13 sites have been assigned to the palustrine system.

Table 5. U.S. Fish and Wildlife Service wetlands inventory codes for selected Lake Traverse Reservation wetlands at which waterquality samples were collected during July 2006.

\begin{tabular}{|c|c|c|c|}
\hline Wetland code & Definition & $\begin{array}{c}\text { Sampling sites }{ }^{1} \\
\text { (fig. 2) }\end{array}$ & $\begin{array}{c}\text { Total number } \\
\text { of sites }\end{array}$ \\
\hline L1UBG & Lacustrine, Limnetic, Unconsolidated Bottom, Intermittently Exposed & S10 & 1 \\
\hline PABF & Palustrine, Aquatic Bed, Semipermanently Flooded & $\mathrm{S} 22$ & 1 \\
\hline PEMF & Palustrine, Emergent, Semipermanently Flooded & S03, S07, S13, S19 & 4 \\
\hline PEMFx & Palustrine, Emergent, Semipermanently Flooded, Excavated & S09 & 1 \\
\hline $\mathrm{PEM} / \mathrm{ABF}$ & Palustrine, Emergent/Aquatic Bed, Semipermanently Flooded & $\mathrm{S} 20, \mathrm{~S} 21$ & 2 \\
\hline PFOC & Palustrine, Forested, Seasonally Flooded & S01 & 1 \\
\hline
\end{tabular}

${ }^{1}$ No wetland inventory code was assigned for sampling sites S15 or S18. 
Manuscript approved for publication, April 20, 2007.

Prepared by the Helena Publishing Service Center.

For more information concerning the research in this report, contact: U.S. Geological Survey

Director, South Dakota Water Science Center

1608 Mountain View Road

Rapid City, SD 57702

http://sd.water.usgs.gov/ 


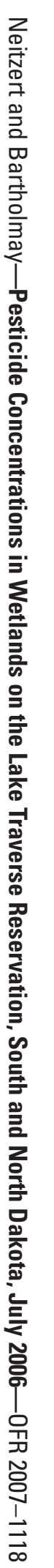

9 Printed on recycled paper 